Supporting Information for:

\title{
Hydroxyl-directed Conjugate Additions of Carbon Nucleophiles to Cyclopentadienones
}

\author{
Anthony J. Pearson* and Ming Zhang \\ Department of Chemistry, Case Western Reserve University, Cleveland, $\mathrm{OH}$ \\ 44106
}




\section{Contents}

Title $\quad$ S1

Contents $\quad$ S2

General Procedures $\quad$ S3

Characterization Data $\quad$ S4

$\begin{array}{ll}\text { NMR Spectra } & \text { S9 }\end{array}$ 


\section{General Procedures:}

All reactions were performed under dry argon atmosphere, in freshly distilled (under nitrogen) solvents. All reagents used are commercially available. Analytical thin-layer chromatography was performed on glass plates precoated with Merck $F_{254}$ silica gel. Visualization was done either with UV light or $5.25 \mathrm{~g}$ PMA / $100 \mathrm{~mL}$ EtOH. Flash chromatography was performed with hexanes and EtOAc mixtures on silica gel with mesh 170-400 under nitrogen pressure. Preparative thin-layer chromatography was run in glass chamber with hexanes and EtOAc mixed solvents. NMR spectra were recorded on a Varian Gemini 200 (200 MHz) or a Varian Gemini 400 (400 MHz) spectrometer. Mass spectra were recorded in-house using a Kratos MS25A instrument.

Silylation of 3-((tert-butyldimethylsilyloxy)methyl)-4-(hydroxymethyl)-2,5-bis(trimethyl silyl)cyclopentadienone (4)

3,4-bis(hydroxymethyl)-2,5-bis(trimethylsilyl)cyclopentadienone (1, $79.3 \mathrm{mg}, 0.28 \mathrm{mmol})$ was dissolved in dry DCM $(11 \mathrm{~mL})$ and cooled to $0{ }^{\circ} \mathrm{C}$. Imidazole $(40.7 \mathrm{mg}, 0.60 \mathrm{mmol})$ and DMAP (1.4 mg, $0.01 \mathrm{mmol}$ ) were added with stirring, followed by injecting DMF $(0.14 \mathrm{~mL})$. After 15 minutes, TBSCl $(45 \mathrm{mg}, 0.29 \mathrm{mmol})$ was added to the reaction mixture, which was then allowed to stand at $0{ }^{\circ} \mathrm{C}$ for $4 \mathrm{~h}$. The reaction was quenched with saturated $\mathrm{NH}_{4} \mathrm{Cl}$ solution, and extracted with DCM $(3 \times 5 \mathrm{~mL})$. The combined organic phase was washed with water $(5 \mathrm{~mL})$ and brine $(5 \mathrm{~mL})$, dried with $\mathrm{Na}_{2} \mathrm{SO}_{4}$, and concentrated. The residue was purified by flash chromatography on silica gel to give $\mathbf{4}$ as a yellow oil $(61.7 \mathrm{mg}, 0.15 \mathrm{mmol}, 83 \%)$ and unreacted $1(26.6 \mathrm{mg}, 34 \%)$.

Addition reactions of 3-((tert-butyldimethylsilyloxy)methyl)-4-(hydroxymethyl)-2,5-bis(trimethylsilyl)cyclopentadienone (4) with Grignard reagents

To a THF $(0.88 \mathrm{~mL})$ solution of $4(10.5 \mathrm{mg}, 0.026 \mathrm{mmol})$ at $-78{ }^{\circ} \mathrm{C}$ was added vinyl $\mathrm{MgBr}(50.0 \mu \mathrm{L} 1 \mathrm{M}$ in THF, $0.05 \mathrm{mmol})$. The reaction mixture was stirred for 15 minutes at $-78{ }^{\circ} \mathrm{C}$. The dry ice-acetone bath was removed and stirring was continued for 1 $\mathrm{h}$ at room temperature. The reaction was quenched with saturated $\mathrm{NH}_{4} \mathrm{Cl}$ solution, and extracted with EtOAc $(3 \times 0.5 \mathrm{~mL})$. The combined organic phase was washed with water and brine, dried with $\mathrm{Na}_{2} \mathrm{SO}_{4}$, evaporated residue and then was purified by preparative TLC to give $\mathbf{6 b}$ as an oil ( $4.3 \mathrm{mg}, 0.015 \mathrm{mmol}, 55 \%)$. 


\section{Characterization Data:}<smiles>CC(C)(C)OCC1=C(CO)C(=O)C([As])=C1CO</smiles>

${ }^{1} \mathrm{H}$ NMR $\left(\mathrm{C}_{6} \mathrm{D}_{6}\right) \delta 4.49(\mathrm{~d}, J=7.6 \mathrm{~Hz}, 2 \mathrm{H}), 4.48(\mathrm{~s}, 2 \mathrm{H}), 3.14(\mathrm{t}, J=7.2 \mathrm{~Hz}, 1 \mathrm{H}), 0.89(\mathrm{~s}$, $9 \mathrm{H}), 0.31(\mathrm{~s}, 9 \mathrm{H}), 0.29(\mathrm{~s}, 9 \mathrm{H}), 0.03(\mathrm{~s}, 6 \mathrm{H})$.

${ }^{13} \mathrm{C} \mathrm{NMR}\left(\mathrm{C}_{6} \mathrm{D}_{6}\right) \delta 215.6,174.9,173.2,136.8,136.5,65.3,63.9,31.2,23.7,5.6,5.5,0.0$ TLC R 0.67 (Hexanes : EtOAc $=5: 1$ ).

HRMS (FAB) $\mathrm{MH}^{+}$calcd. for $\mathrm{C}_{19} \mathrm{H}_{39} \mathrm{O}_{3} \mathrm{Si}_{3} 399.2207$ found 399.2217.

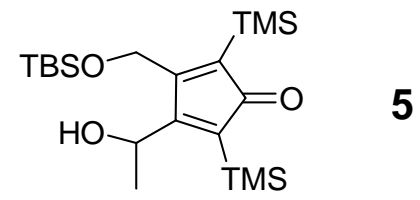

${ }^{1} \mathrm{H}$ NMR $\left(\mathrm{C}_{6} \mathrm{D}_{6}\right) \delta 5.11(\mathrm{dq}, J=8.8,6.8 \mathrm{~Hz}, 1 \mathrm{H}), 4.75$ and $4.52(\mathrm{ABq}, J=12.8 \mathrm{~Hz}, 2 \mathrm{H})$, $3.85(\mathrm{~d}, J=8.8 \mathrm{~Hz}, 1 \mathrm{H}), 1.46(\mathrm{~d}, J=6.8 \mathrm{~Hz}, 3 \mathrm{H}), 0.91(\mathrm{~s}, 9 \mathrm{H}), 0.31(\mathrm{~s}, 9 \mathrm{H}), 0.29(\mathrm{~s}, 9 \mathrm{H})$, $0.06(\mathrm{~s}, 3 \mathrm{H}), 0.05(\mathrm{~s}, 3 \mathrm{H})$.

${ }^{13} \mathrm{C}$ NMR $\left(\mathrm{C}_{6} \mathrm{D}_{6}\right) \delta 210.1,172.8,166.4,132.6,129.0,65.9,60.7,25.9,23.7,18.4,0.38$, $0.27,-5.16,-5.19$.

TLC $\mathrm{R}_{\mathrm{f}} 0.65$ (Hexanes : EtOAc $=5: 1$ ).

HRMS (EI) $\mathrm{M}^{+}$calcd. for $\mathrm{C}_{20} \mathrm{H}_{40} \mathrm{O}_{3} \mathrm{Si}_{3} 412.2285$ found 412.2191, $\mathrm{M}^{+}-\mathrm{CH}_{3}$ calcd. for $\mathrm{C}_{19} \mathrm{H}_{37} \mathrm{O}_{3} \mathrm{Si}_{3} 397.2051$ found 397.2013, $\mathrm{M}^{+}-\mathrm{C}_{4} \mathrm{H}_{9}$ calcd. for $\mathrm{C}_{16} \mathrm{H}_{31} \mathrm{O}_{3} \mathrm{Si}_{3} 355.1583$ found 355.1576 .

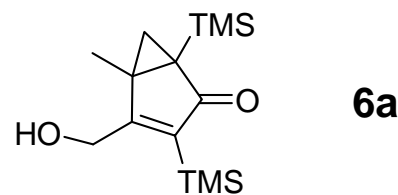

${ }^{1} \mathrm{H}$ NMR $\left(\mathrm{C}_{6} \mathrm{D}_{6}\right) \delta 4.27$ and $3.98(\mathrm{ABq}, J=13.0 \mathrm{~Hz}, 2 \mathrm{H}), 1.32(\mathrm{~s}, 3 \mathrm{H}), 1.13$ and 1.05 $(\mathrm{ABq}, J=2.6 \mathrm{~Hz}, 2 \mathrm{H}), 0.29$ (s, 9H), 0.25 (s, 9H).

${ }^{13} \mathrm{C}$ NMR $\left(\mathrm{C}_{6} \mathrm{D}_{6}\right) \delta 211.9,185.3,133.4,59.1,44.4,37.7,29.9,14.3,0.2,-0.7$

TLC $\mathrm{R}_{\mathrm{f}} 0.34$ (Hexanes : EtOAc $=5: 1$ ).

HRMS (FAB) $\mathrm{MH}^{+}$calcd. for $\mathrm{C}_{14} \mathrm{H}_{27} \mathrm{O}_{2} \mathrm{Si}_{2} 283.1549$ found 283.1546.<smiles>CC(C)(C)OCC1=C([As])C(C)(O)C([Y15](=O)[O-])=C1CO</smiles> 
${ }^{1} \mathrm{H}$ NMR $\left(\mathrm{C}_{6} \mathrm{D}_{6}\right) \delta 4.52$ and $4.29(\mathrm{ABq}, J=11.6 \mathrm{~Hz}, 2 \mathrm{H}), 4.48$ and $4.34(\mathrm{dABq}, J=4.4$ and 8.0, 2H), $3.31(\mathrm{dd}, J=4.4$ and $12.8 \mathrm{~Hz}, 1 \mathrm{H}), 1.44(\mathrm{~s}, 3 \mathrm{H}), 0.94(\mathrm{~s}, 9 \mathrm{H}), 0.36(\mathrm{~s}, 9 \mathrm{H})$, $0.34(\mathrm{~s}, 9 \mathrm{H}), 0.11(\mathrm{~s}, 3 \mathrm{H}), 0.10(\mathrm{~s}, 3 \mathrm{H})$.

${ }^{13} \mathrm{C}$ NMR $\left(\mathrm{C}_{6} \mathrm{D}_{6}\right) \delta 155.0,154.9,151.3,149.8,92.8,59.0,57.3,24.8,21.9,17.2,0.1,0.0$, - 6.5, - 6.6.

TLC R 0.57 (Hexanes : EtOAc $=5: 1$ ).

HRMS (FAB) Both EI and FAB failed to obtain $\mathrm{M}^{+}$.

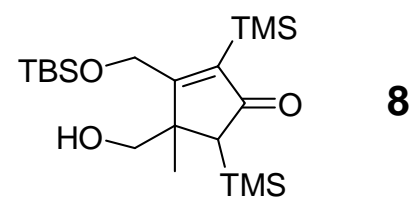

${ }^{1} \mathrm{H}$ NMR $\left(\mathrm{C}_{6} \mathrm{D}_{6}\right) \delta 4.45$ and $4.17(\mathrm{ABq}, J=11.4 \mathrm{~Hz}, 2 \mathrm{H}), 3.40(\mathrm{~m}, 2 \mathrm{H}), 2.36(\mathrm{~s}, 1 \mathrm{H}), 1.18$ $(\mathrm{s}, 3 \mathrm{H}), 0.93(\mathrm{~s}, 9 \mathrm{H}), 0.38(\mathrm{~s}, 9 \mathrm{H}), 0.20(\mathrm{~s}, 9 \mathrm{H}), 0.06(\mathrm{~s}, 3 \mathrm{H}), 0.04(\mathrm{~s}, 3 \mathrm{H})$.

${ }^{13} \mathrm{C}$ NMR $\left(\mathrm{C}_{6} \mathrm{D}_{6}\right) \delta 217.4,187.7,150.8,76.4,64.1,60.2,56.9,31.5,25.6,23.9,5.6,5.3$, $0.2,0.0$.

TLC $\mathrm{R}_{\mathrm{f}} 0.30$ (Hexanes : EtOAc $=5: 1$ ).

HRMS (FAB) $\mathrm{MH}^{+}$calcd. for $\mathrm{C}_{20} \mathrm{H}_{43} \mathrm{O}_{3} \mathrm{Si}_{3} 415.2520$, found 415.2513.

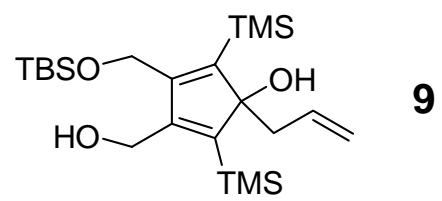

${ }^{1} \mathrm{H}$ NMR $\left(\mathrm{C}_{6} \mathrm{D}_{6}\right) \delta 5.41(\mathrm{~m}, 1 \mathrm{H}), 4.98$ and $4.92(\mathrm{dABq}, J=1.6$ and $17.2 \mathrm{~Hz}, 2 \mathrm{H}), 4.51$ and $4.31(\mathrm{ABq}, J=12 \mathrm{~Hz}, 2 \mathrm{H}), 4.50$ and $4.35(\mathrm{dABq}, J=4$ and $13.6 \mathrm{~Hz}, 2 \mathrm{H}), 3.27(\mathrm{dd}, J=8$ and $4.4 \mathrm{~Hz}, 1 \mathrm{H}), 2.85(\mathrm{dd}, J=2$ and $6.8 \mathrm{~Hz}, 2 \mathrm{H}), 0.93(\mathrm{~s}, 9 \mathrm{H}), 0.35(\mathrm{~s}, 9 \mathrm{H}), 0.33(\mathrm{~s}, 9 \mathrm{H})$, $0.10(\mathrm{~s}, 3 \mathrm{H}), 0.09$ (s, 3H).

${ }^{13} \mathrm{C}$ NMR $\left(\mathrm{C}_{6} \mathrm{D}_{6}\right) \delta 154.5,154.1,154.0,133.3,117.0,96.3,59.8,58.2,40.0,25.8,18.2$, $1.0,0.9,-5.5,-5.6$.

TLC $\mathrm{R}_{\mathrm{f}} 0.54$ (Hexanes : EtOAc $=5: 1$ ).

HRMS (FAB) $\mathrm{MH}^{+}$calcd. for $\mathrm{C}_{22} \mathrm{H}_{45} \mathrm{O}_{3} \mathrm{Si}_{3} 441.2676$, found 441.2607, $\mathrm{M}^{+}-\mathrm{H}$ calcd. for $\mathrm{C}_{22} \mathrm{H}_{43} \mathrm{O}_{3} \mathrm{Si}_{3}$ 439.2520, found 439.2509, $\mathrm{M}^{+}-\mathrm{OH}$ calcd. for $\mathrm{C}_{22} \mathrm{H}_{43} \mathrm{O}_{2} \mathrm{Si}_{3}$ 423.2571, found 423.2451 .<smiles>[Y9]C12CC1(C=C)C(CO)=C([Y5](=O)[O-])C2=O</smiles>

${ }^{1} \mathrm{H}$ NMR $\left(\mathrm{C}_{6} \mathrm{D}_{6}\right) \delta 5.80(\mathrm{dd}, J=10.0$ and $17.6 \mathrm{~Hz}, 1 \mathrm{H}), 4.97(\mathrm{~m}, 2 \mathrm{H}), 4.31$ and 4.10 $(\mathrm{dABq}, J=6.8$ and $13.4 \mathrm{~Hz}, 2 \mathrm{H}), 1.39$ and $1.24(\mathrm{ABq}, J=2.8 \mathrm{~Hz}, 2 \mathrm{H}), 0.29(\mathrm{~s}, 9 \mathrm{H}), 0.21$ $(\mathrm{s}, 9 \mathrm{H})$.

${ }^{13} \mathrm{C}$ NMR $\left(\mathrm{C}_{6} \mathrm{D}_{6}\right) \delta 210.9,183.9,134.4,133.9,119.7,59.4,44.2,40.9,31.6,-0.2,-1.2$. 
TLC $\mathrm{R}_{\mathrm{f}} 0.38$ (Hexanes : EtOAc $=5: 1$ ).

HRMS (FAB) $\mathrm{MH}^{+}$calcd. for $\mathrm{C}_{15} \mathrm{H}_{27} \mathrm{O}_{2} \mathrm{Si}_{2} 295.1549$, found 295.1546.<smiles>C=C1CC(=O)C(CO)=C1CO</smiles>

${ }^{1} \mathrm{H}$ NMR $\left(\mathrm{C}_{6} \mathrm{D}_{6}\right) \delta 5.15(\mathrm{t}, J=1.6 \mathrm{~Hz}, 1 \mathrm{H}), 4.74(\mathrm{t}, J=1.6 \mathrm{~Hz}, 1 \mathrm{H}), 4.11(\mathrm{~d}, J=3.6 \mathrm{~Hz}$, $2 \mathrm{H}), 2.66(\mathrm{t}, J=1.6 \mathrm{~Hz}, 2 \mathrm{H}), 0.70(\mathrm{t}, J=3.6 \mathrm{~Hz}, 1 \mathrm{H}), 0.33(\mathrm{~s}, 9 \mathrm{H})$.

${ }^{13} \mathrm{C}$ NMR $\left(\mathrm{C}_{6} \mathrm{D}_{6}\right) \delta 207.8,174.4,146.8,145.4,109.1,57.5,41.3,0.0$.

TLC $\mathrm{R}_{\mathrm{f}} 0.13$ (Hexanes : EtOAc $=5: 1$ ).

HRMS (FAB) $\mathrm{MH}^{+}$calcd. for $\mathrm{C}_{10} \mathrm{H}_{17} \mathrm{O}_{2} \mathrm{Si}$ 197.0998, found 197.1008.

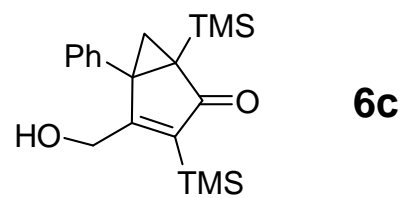

${ }^{1} \mathrm{H}$ NMR $\left(\mathrm{C}_{6} \mathrm{D}_{6}\right) \delta 7.05(5 \mathrm{H}), 4.21$ and $3.87(\mathrm{dABq}, J=5.6$ and $13.4 \mathrm{~Hz}, 2 \mathrm{H}), 1.67$ and $1.46(\mathrm{ABq}, J=2.8 \mathrm{~Hz}, 2 \mathrm{H}), 0.34$ (s, 9H), -0.04 (s, 9H).

${ }^{13} \mathrm{C}$ NMR $\left(\mathrm{CDCl}_{3}\right) \delta 215.0,187.0,138.9,135.8,130.0,61.6,48.7,43.1,34.4,2.0,0.0$

TLC $\mathrm{R}_{\mathrm{f}} 0.36$ (Hexanes : EtOAc $=5: 1$ ).

HRMS (FAB) $\mathrm{MH}^{+}$calcd. for $\mathrm{C}_{19} \mathrm{H}_{29} \mathrm{O}_{2} \mathrm{Si}_{2}$ 345.1706, found 345.1696, $\mathrm{M}^{+}$- OH calcd. for $\mathrm{C}_{19} \mathrm{H}_{27} \mathrm{OSi}_{2}$ 327.1601, found 327.1309, $\mathrm{M}^{+}-\mathrm{CH}_{3}$ calcd. for $\mathrm{C}_{18} \mathrm{H}_{25} \mathrm{O}_{2} \mathrm{Si}_{2}$ 329.1394, found 329.1326 .<smiles>CC(O)C1=C([As])C(=O)C(C)(C)C1(C)C</smiles>

${ }^{1} \mathrm{H}$ NMR $\left(\mathrm{C}_{6} \mathrm{D}_{6}\right) \delta 4.74(\mathrm{q}, J=6.4 \mathrm{~Hz}, 1 \mathrm{H}), 1.47(\mathrm{~s}, 3 \mathrm{H}), 1.22(\mathrm{~d}, J=6.4 \mathrm{~Hz}, 3 \mathrm{H}), 1.03$ and $1.00(\mathrm{ABq}, J=2.4 \mathrm{~Hz}, 2 \mathrm{H}), 0.269(\mathrm{~s}, 9 \mathrm{H}), 0.267(\mathrm{~s}, 9 \mathrm{H})$.

${ }^{13} \mathrm{C}$ NMR $\left(\mathrm{C}_{6} \mathrm{D}_{6}\right) \delta 211.9,188.0,132.7,67.4,45.0,37.7,30.1,22.3,16.4,0.21,-0.84$.

TLC $\mathrm{R}_{\mathrm{f}} 0.32$ (Hexanes : EtOAc $=5: 1$ ).

HRMS (EI) M calcd. for $\mathrm{C}_{15} \mathrm{H}_{28} \mathrm{O}_{2} \mathrm{Si}_{2} 296.1628$, found 296.1629, $\mathrm{M}^{+}-\mathrm{CH}_{3}$ calcd. for $\mathrm{C}_{14} \mathrm{H}_{25} \mathrm{O}_{2} \mathrm{Si}_{2} 281.1394$, found 281.1376 .<smiles>CCCOCC1=C(C(C)O)C(COCC)=C(C(C)C)C1(C)O</smiles>

${ }^{1} \mathrm{H}$ NMR $\left(\mathrm{C}_{6} \mathrm{D}_{6}\right) \delta 5.04(\mathrm{dq}, J=9.6$ and $6.8 \mathrm{~Hz}, 1 \mathrm{H}), 4.73$ and $4.47(\mathrm{ABq}, J=12 \mathrm{~Hz}, 2 \mathrm{H})$, $4.35(\mathrm{~d}, J=9.6 \mathrm{~Hz}, 1 \mathrm{H}), 1.49(\mathrm{~d}, J=6.8 \mathrm{~Hz}, 3 \mathrm{H}), 0.96(\mathrm{~s}, 9 \mathrm{H}), 0.36(\mathrm{~s}, 9 \mathrm{H}), 0.35(\mathrm{~s}, 9 \mathrm{H})$, $0.14(\mathrm{~s}, 3 \mathrm{H}), 0.12(\mathrm{~s}, 3 \mathrm{H})$. 
${ }^{13} \mathrm{C}$ NMR $\left(\mathrm{C}_{6} \mathrm{D}_{6}\right)$ not available due to very small sample.

TLC R $\mathrm{R}_{\mathrm{f}} 0.38$ (Hexanes : EtOAc $=5: 1$ ).

HRMS (EI) $\mathrm{M}^{+}$calcd. for $\mathrm{C}_{21} \mathrm{H}_{44} \mathrm{O}_{3} \mathrm{Si}_{3}$ 428.2598, found 428.2555, $\mathrm{M}^{+}-\mathrm{H}_{2} \mathrm{O}$ calcd. for $\mathrm{C}_{21} \mathrm{H}_{42} \mathrm{O}_{2} \mathrm{Si}_{3}$ 410.2493, found 410.2482.

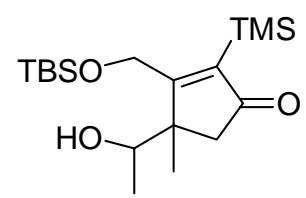

13

${ }^{1} \mathrm{H}$ NMR $\left(\mathrm{C}_{6} \mathrm{D}_{6}\right) \delta 4.37$ and $4.08(\mathrm{ABq}, J=11.2 \mathrm{~Hz}, 2 \mathrm{H}), 3.84(\mathrm{dq}, J=4.8$ and $6.4 \mathrm{~Hz}$, $1 \mathrm{H}), 2.70$ and $1.89(\mathrm{ABq}, J=17.6 \mathrm{~Hz}, 2 \mathrm{H}), 2.69(\mathrm{~d}, J=4.8 \mathrm{~Hz}, 1 \mathrm{H}), 0.98(\mathrm{~d}, J=6.4 \mathrm{~Hz}$, $3 \mathrm{H}), 0.89$ (s, 9H), 0.85 (s, 3H), 0.36 (s, 9H), 0.02 (s, 3H), 0.00 (s, 3H).

${ }^{13} \mathrm{C}$ NMR $\left(\mathrm{C}_{6} \mathrm{D}_{6}\right) \delta 210.6,183.9,144.7,70.2,58.3,53.8,45.2,25.7,23.3,18.1,18.0,-0.2$, $-5.6,-5.8$.

TLC $\mathrm{R}_{\mathrm{f}} 0.15$ (Hexanes : EtOAc $=5: 1$ ).

HRMS (EI) $\mathrm{MH}^{+}$calcd. for $\mathrm{C}_{18} \mathrm{H}_{37} \mathrm{O}_{3} \mathrm{Si}_{2}$ 356.2203, found 356.2206.<smiles>C=CCC1(O)C(COC)=C(C(C)O)C(COC(C)(C)C)=C1[Y15](=O)[Na]</smiles>

14 1st diastereomer

${ }^{1} \mathrm{H}$ NMR $\left(\mathrm{C}_{6} \mathrm{D}_{6}\right) \delta 5.52(\mathrm{~m}, 1 \mathrm{H}), 4.97(\mathrm{~m}, 2 \mathrm{H}), 4.96(\mathrm{dq}, J=8.8$ and $6.8 \mathrm{~Hz}, 1 \mathrm{H}), 4.66$ and $4.55(\mathrm{ABq}, J=11.6 \mathrm{~Hz}, 2 \mathrm{H}), 3.84(\mathrm{~d}, J=8.8 \mathrm{~Hz}, 1 \mathrm{H}), 2.86(\mathrm{~m}, 2 \mathrm{H}), 1.54(\mathrm{~d}, J=6.8$ $\mathrm{Hz}, 3 \mathrm{H}), 0.95$ (s, 9H), 0.33 (s, 9H), 0.32 (s, 9H), 0.13 (s, 3H), 0.11 (s, 3H).

${ }^{13} \mathrm{C}$ NMR $\left(\mathrm{C}_{6} \mathrm{D}_{6}\right) \delta 158.2,155.9,152.1,151.4,133.4,116.9,95.7,66.3,60.7,40.0,25.8$, $23.8,18.2,1.12,1.11,-5.34,-5.37$.

TLC R 0.29 (Hexanes : EtOAc $=10: 1$ ).

HRMS (EI) $\mathrm{M}^{+}$calcd. for $\mathrm{C}_{23} \mathrm{H}_{46} \mathrm{O}_{3} \mathrm{Si}_{3} 454.2755$, found 454.2757.<smiles>C=CCC1(O)C([Y15](=O)[O-])=C(COC(C)(C)C)C(C(C)O)=C1C(C)C</smiles>

14 2nd diastereomer

${ }^{1} \mathrm{H}$ NMR $\left(\mathrm{C}_{6} \mathrm{D}_{6}\right) \delta 5.32(\mathrm{~m}, 1 \mathrm{H}), 5.00(\mathrm{~m}, 2 \mathrm{H}), 4.94(\mathrm{dq}, J=12$ and $6.8 \mathrm{~Hz}, 1 \mathrm{H}), 4.69$ and $4.43(\mathrm{ABq}, J=12 \mathrm{~Hz}, 2 \mathrm{H}), 4.43(\mathrm{~d}, J=12 \mathrm{~Hz}, 1 \mathrm{H}), 2.82(\mathrm{~d}, J=7.2,2 \mathrm{H}), 1.48$ (d, $J=6.8$ $\mathrm{Hz}, 3 \mathrm{H}), 0.96$ (s, 9H), 0.34 (s, 9H), 0.33 (s, 9H), 0.13 (s, 3H), 0.11 (s, 3H).

${ }^{13} \mathrm{C}$ NMR $\left(\mathrm{C}_{6} \mathrm{D}_{6}\right) \delta 157.9,155.7,151.9,151.2,133.2,116.8,95.3,66.1,60.6,40.0,25.8$, 24.0, 18.2, 1.13, 0.99, -5.4, -5.5.

TLC R 0.21 (Hexanes : EtOAc $=10: 1$ ).

HRMS (EI) $\mathrm{M}^{+}$calcd. for $\mathrm{C}_{23} \mathrm{H}_{46} \mathrm{O}_{3} \mathrm{Si}_{3}$ 454.2755, found 454.2766. 


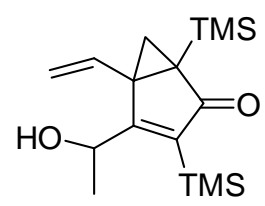

\section{$11 b$}

${ }^{1} \mathrm{H}$ NMR $\left(\mathrm{C}_{6} \mathrm{D}_{6}\right) \delta 6.20$ and $6.16(\mathrm{ABq}, J=10 \mathrm{~Hz}, 1 \mathrm{H}), 4.89(\mathrm{~m}, 2 \mathrm{H}), 4.79(\mathrm{q}, J=6.4 \mathrm{~Hz}$, $1 \mathrm{H}), 1.50$ and $1.10(\mathrm{ABq}, J=2.8 \mathrm{~Hz}, 2 \mathrm{H}), 1.22(\mathrm{~d}, J=6.4 \mathrm{~Hz}, 3 \mathrm{H}), 0.27(\mathrm{~s}, 9 \mathrm{H}), 0.22(\mathrm{~s}$, 9H).

${ }^{13} \mathrm{C}$ NMR $\left(\mathrm{C}_{6} \mathrm{D}_{6}\right) \delta 211.2,186.8,135.4,133.2,118.3,67.8,44.2,40.2,31.3,23.0,0.01,-$ 0.91 .

TLC $\mathrm{R}_{\mathrm{f}} 0.51$ (Hexanes : EtOAc $=5: 1$ ).

HRMS (FAB) $\mathrm{MH}^{+}$calcd. for $\mathrm{C}_{16} \mathrm{H}_{29} \mathrm{O}_{2} \mathrm{Si}_{2}$ 309.1706, found 309.1694, $\mathrm{M}^{+}$- OH calcd. for $\mathrm{C}_{16} \mathrm{H}_{27} \mathrm{OSi}_{2} 291.1601$, found 291.1574.<smiles>CC(O)C1=C([N+](=O)[O-])C2(C)CCC1(c1ccccc1)C(=O)C2=O</smiles>

${ }^{1} \mathrm{H}$ NMR $\left(\mathrm{C}_{6} \mathrm{D}_{6}\right) \delta 6.90$ and $7.40(5 \mathrm{H}), 4.77(\mathrm{q}, J=6.8 \mathrm{~Hz}, 1 \mathrm{H}), 1.61$ and $1.32(\mathrm{ABq}, J=$ $3.2 \mathrm{~Hz}, 2 \mathrm{H}), 1.15(\mathrm{~d}, J=6.8 \mathrm{~Hz}, 3 \mathrm{H}), 0.37(\mathrm{~s}, 9 \mathrm{H}),-0.05(\mathrm{~s}, 9 \mathrm{H})$.

${ }^{13} \mathrm{C}$ NMR $\left(\mathrm{C}_{6} \mathrm{D}_{6}\right) \delta 211.8,187.2,137.6,133.4,132.1,129.6,128.8,68.2,46.7,40.8,32.1$, $23.4,0.22,-2.19$.

${ }^{13} \mathrm{C}$ NMR $\left(\mathrm{CD}_{3} \mathrm{OD}\right) \delta 215.8,191.9,138.4,134.1,133.6,131.1,129.7,128.8,128.4,68.3$, $43.4,33.4,22.7,0.56,-2.04$.

TLC $\mathrm{R}_{\mathrm{f}} 0.52$ (Hexanes : EtOAc $=5: 1$ ).

HRMS (EI) $\mathrm{M}^{+}$calcd. for $\mathrm{C}_{20} \mathrm{H}_{30} \mathrm{O}_{2} \mathrm{Si}_{2}$ 358.1784, found 358.1782. 
NMR Spectra:

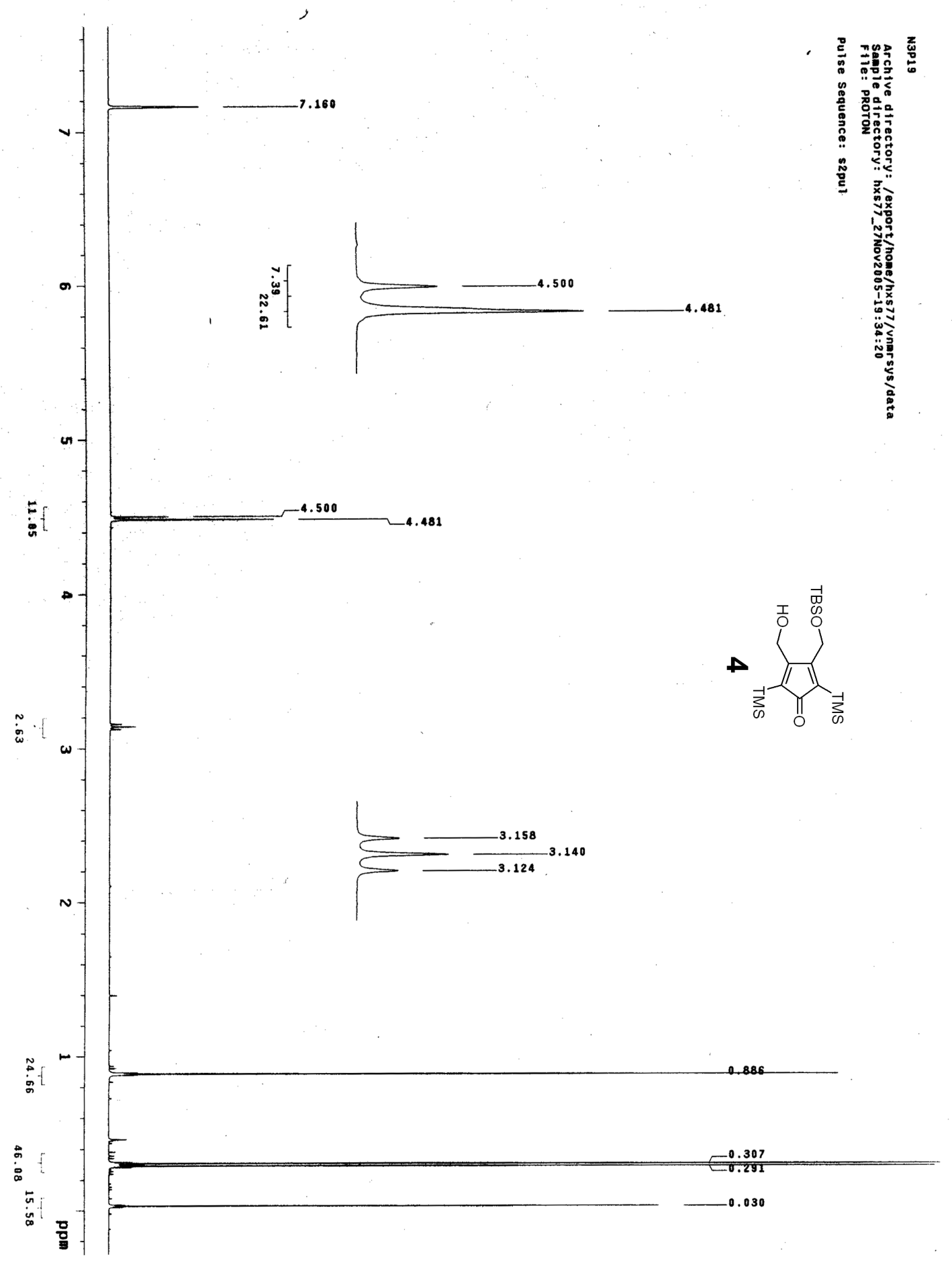




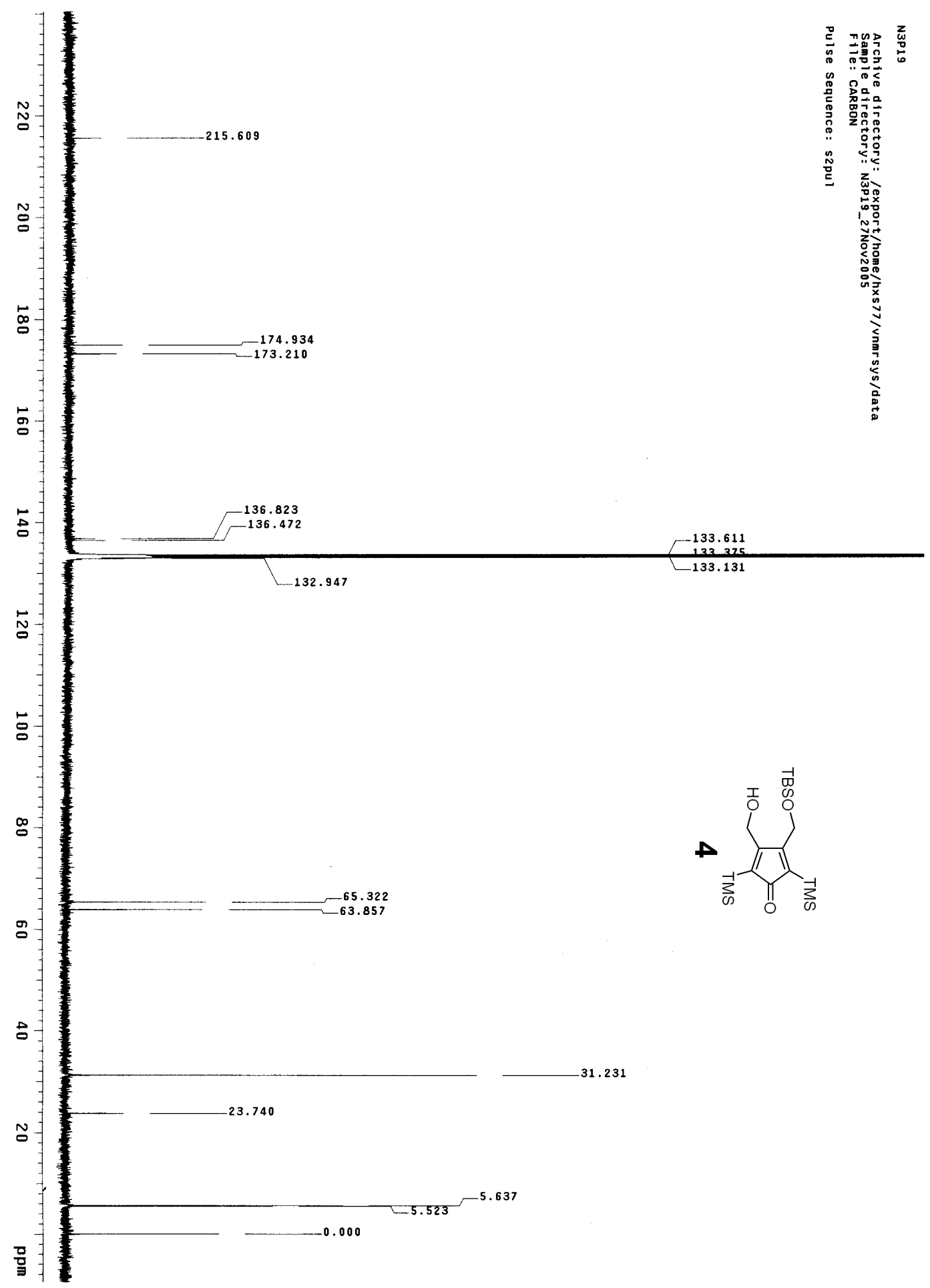




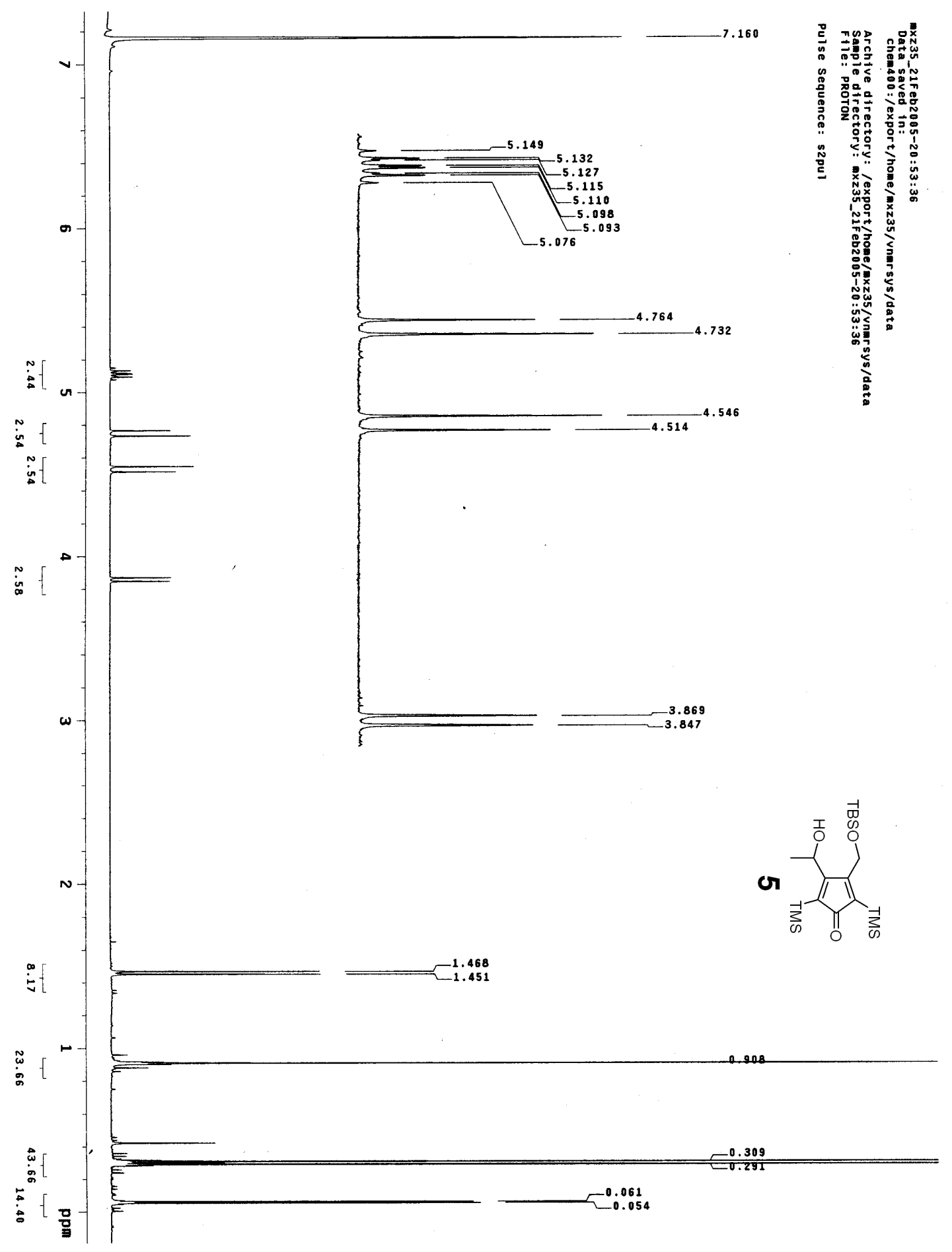




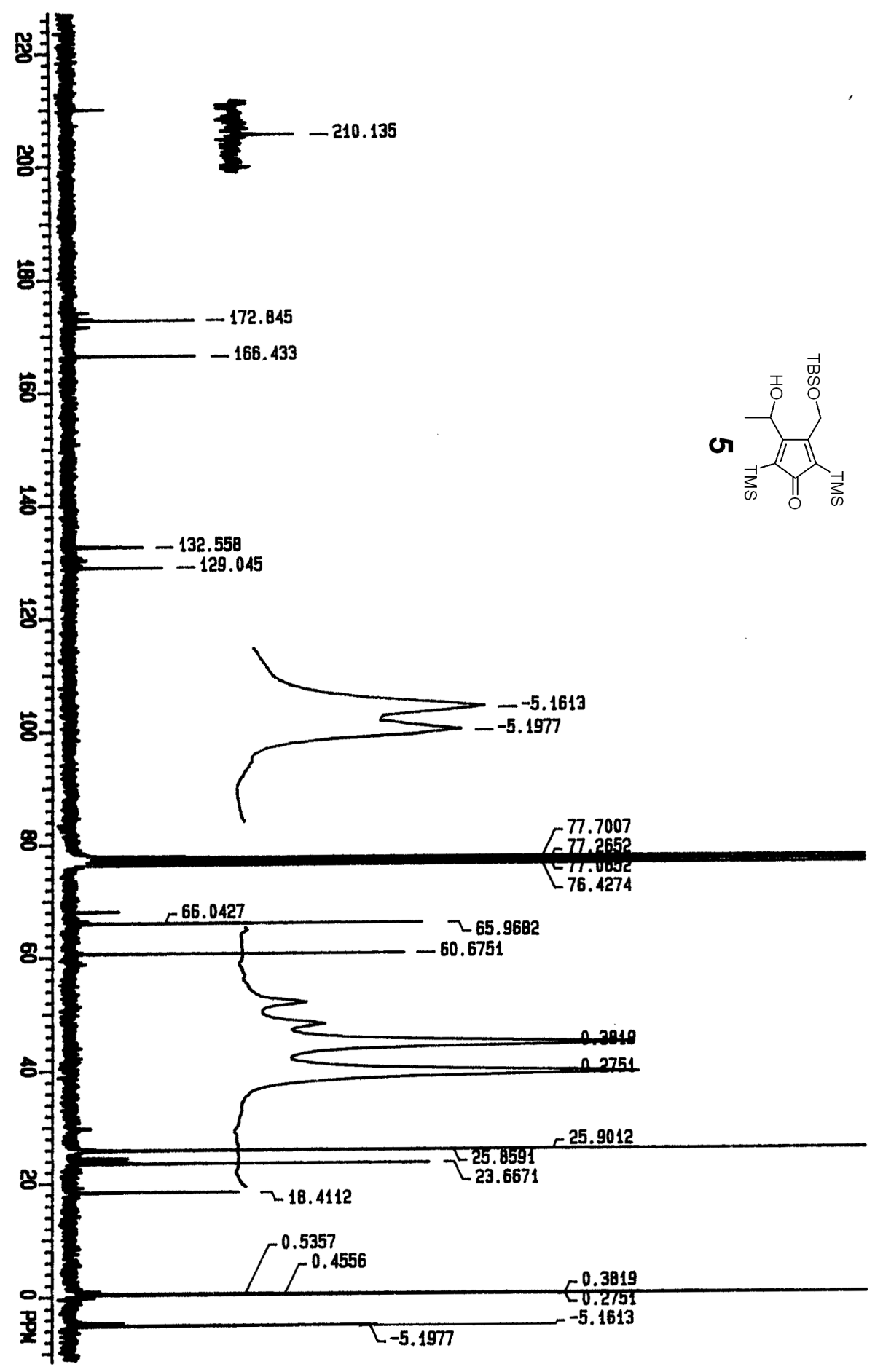




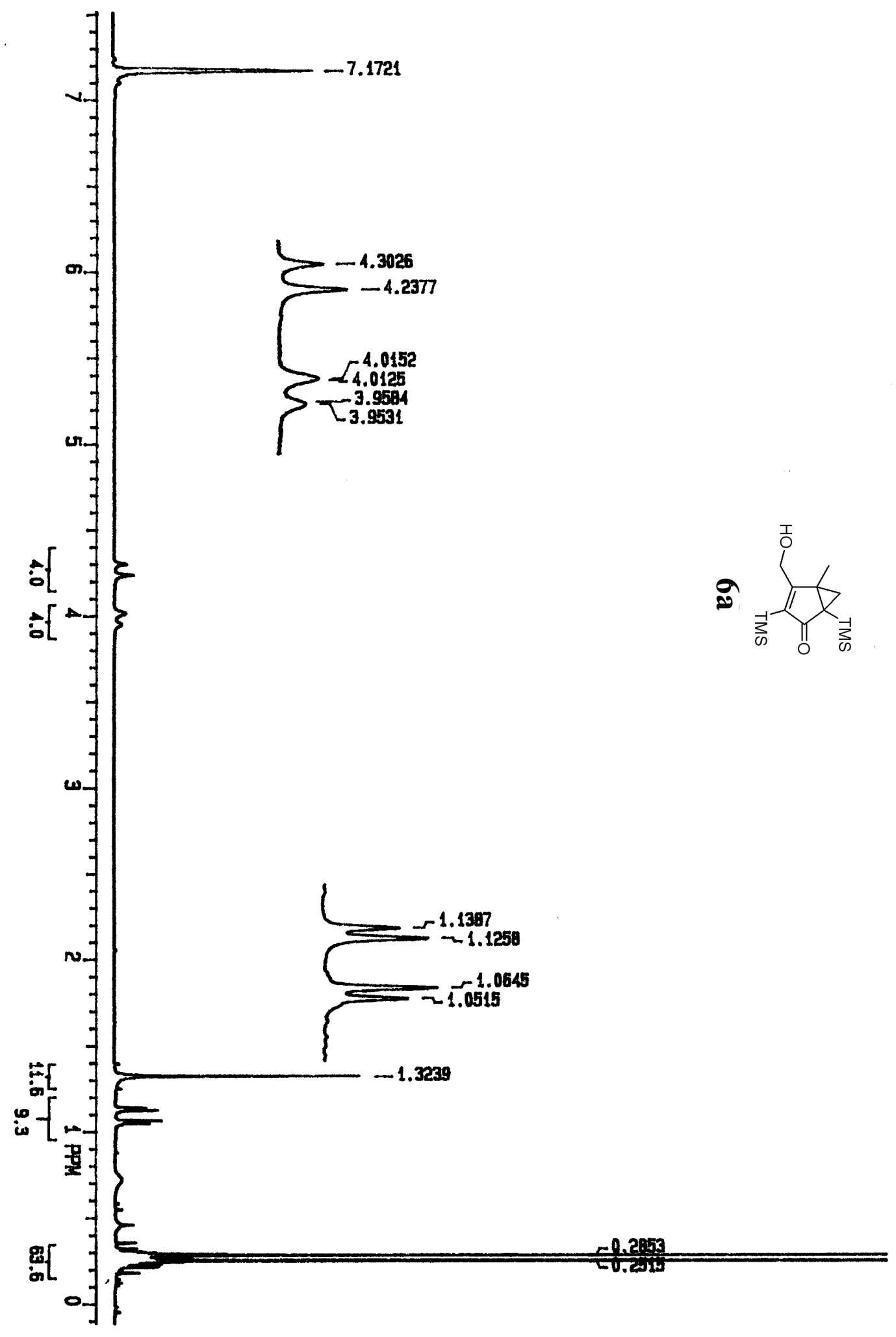




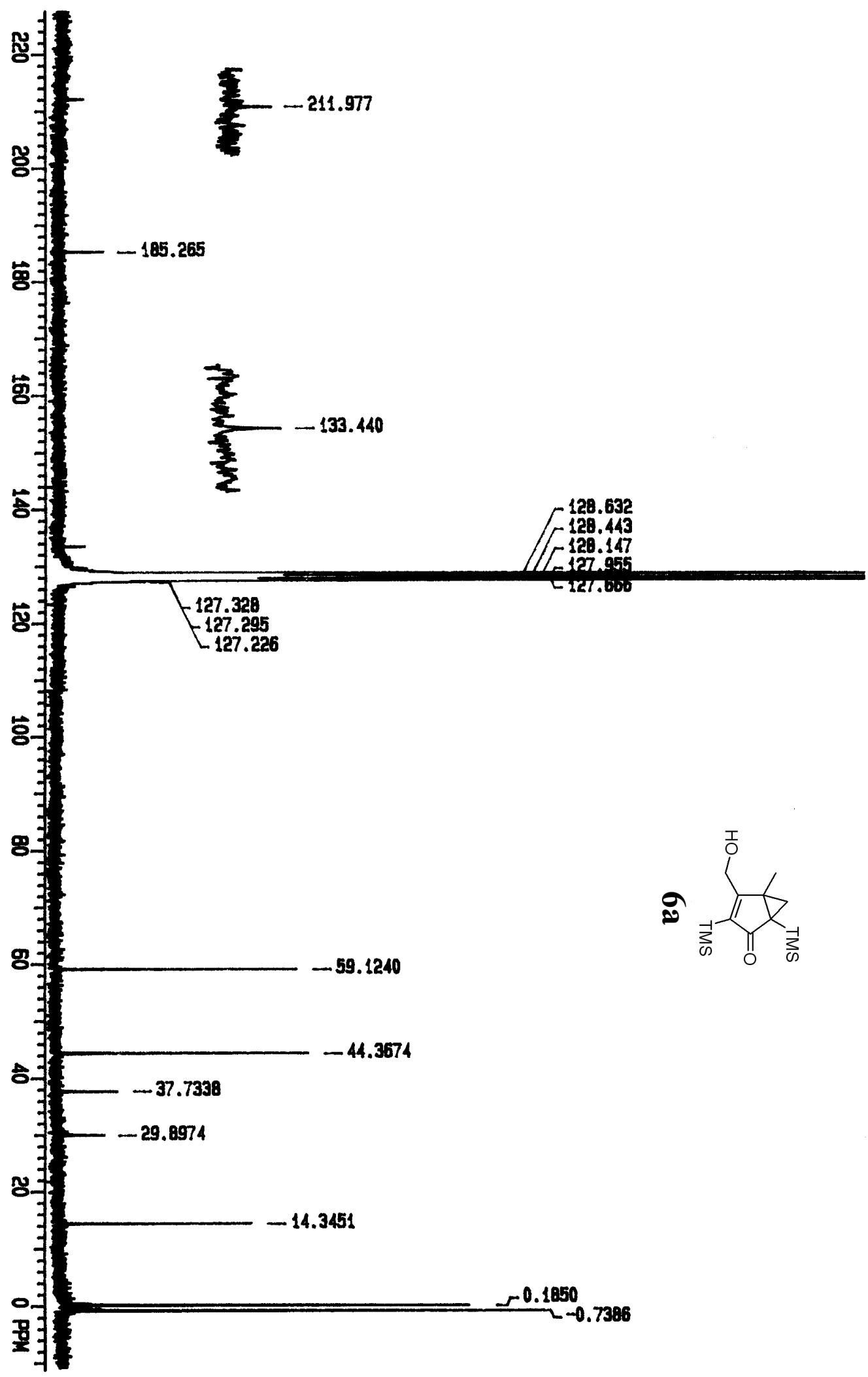




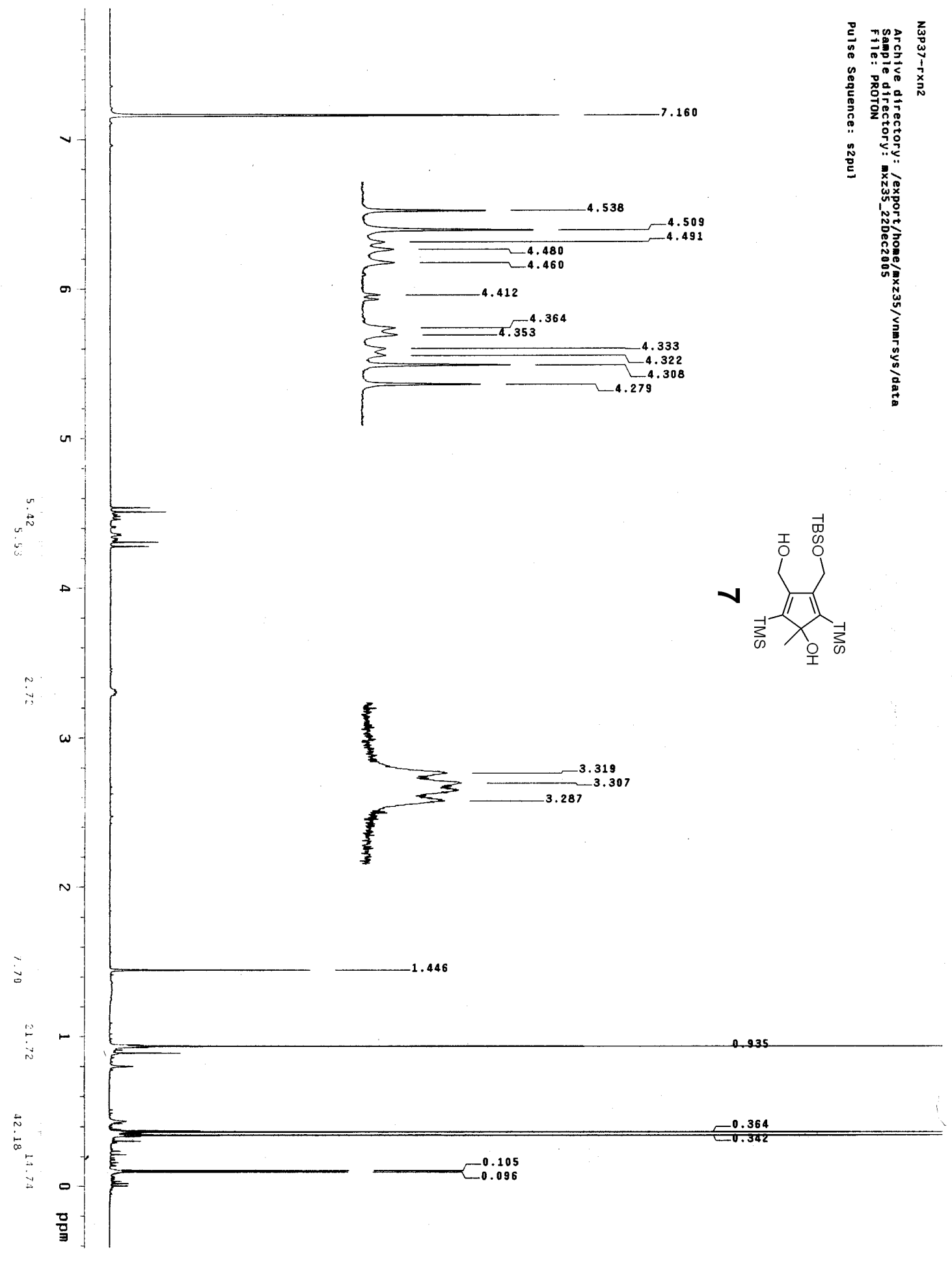




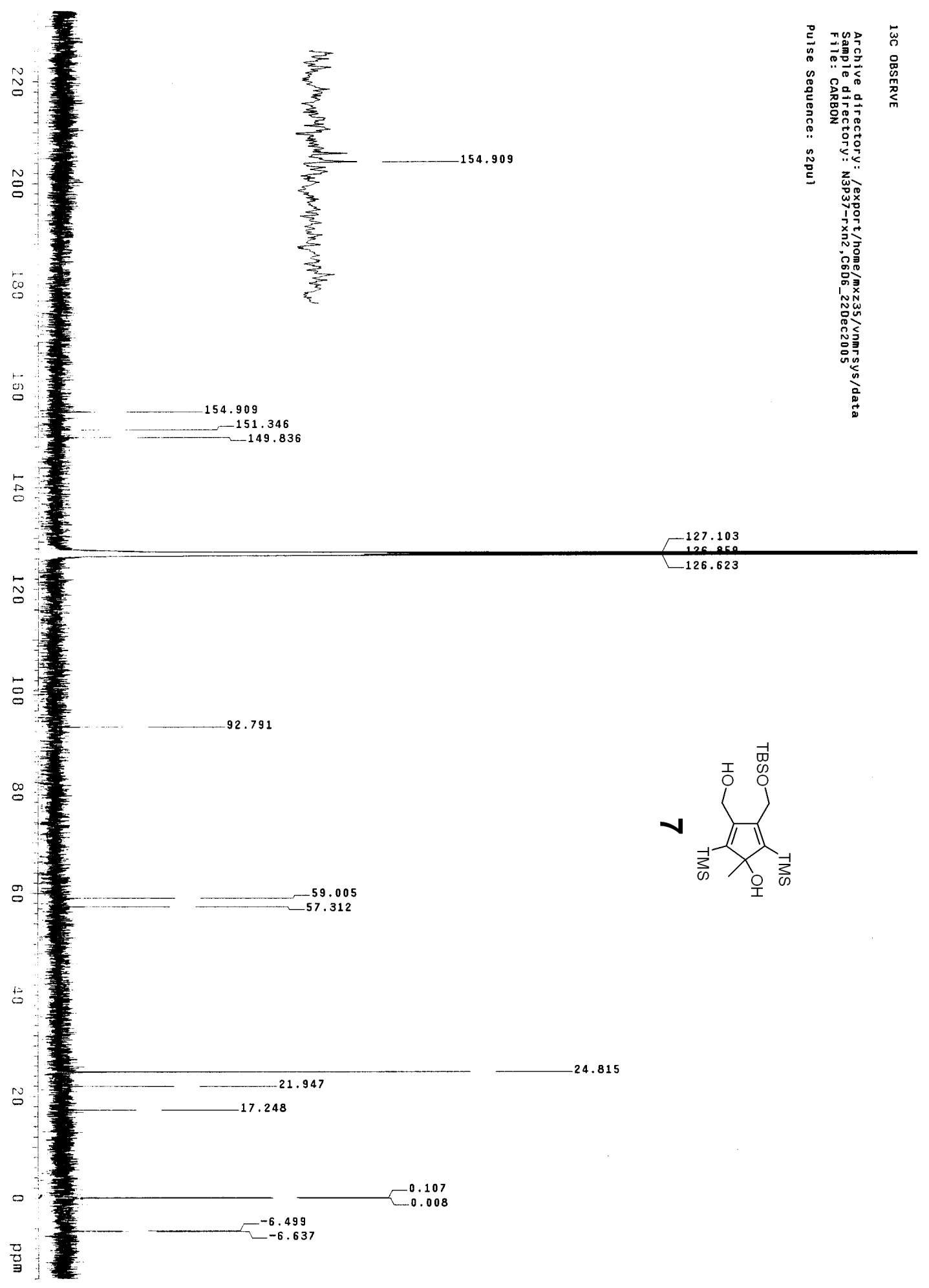




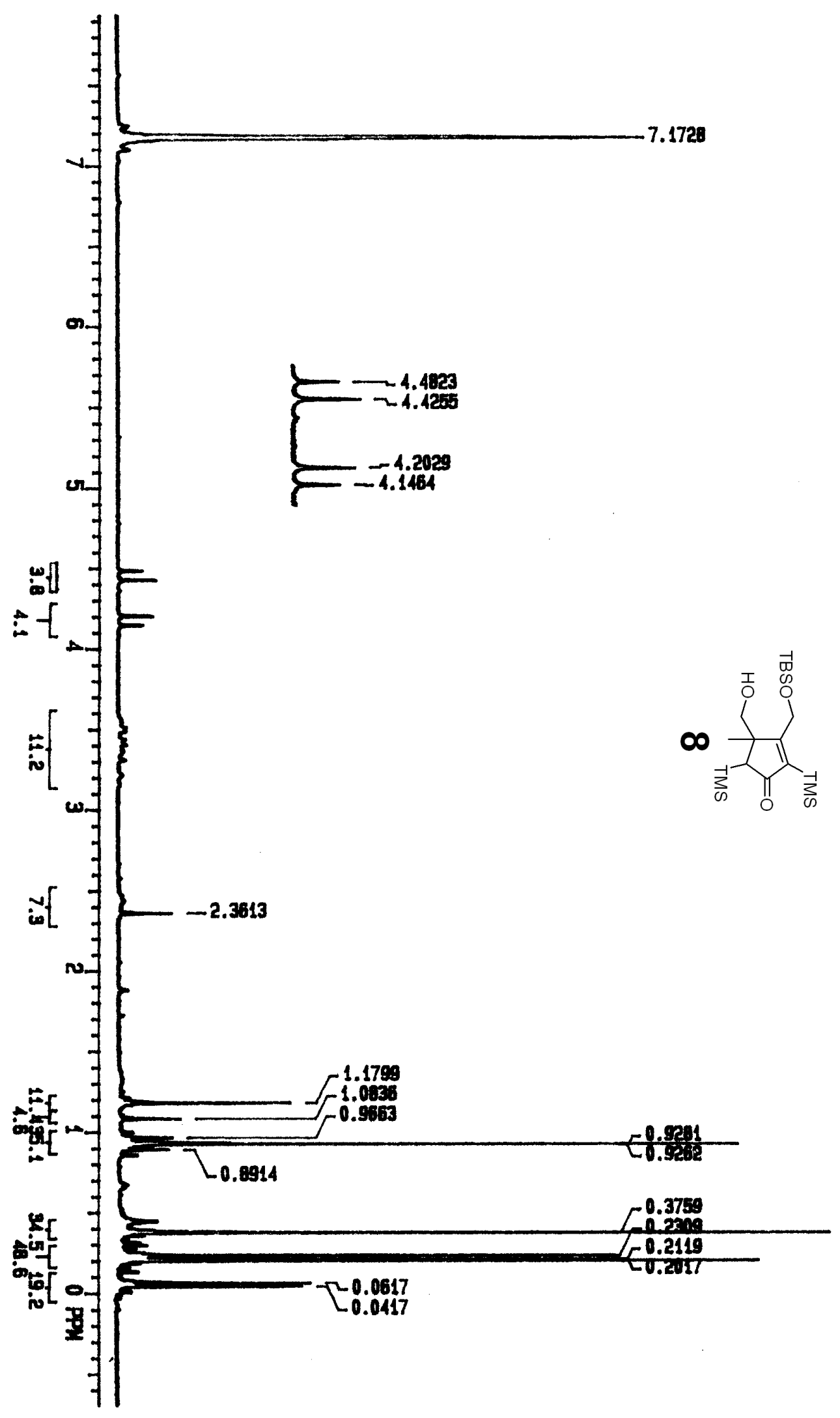




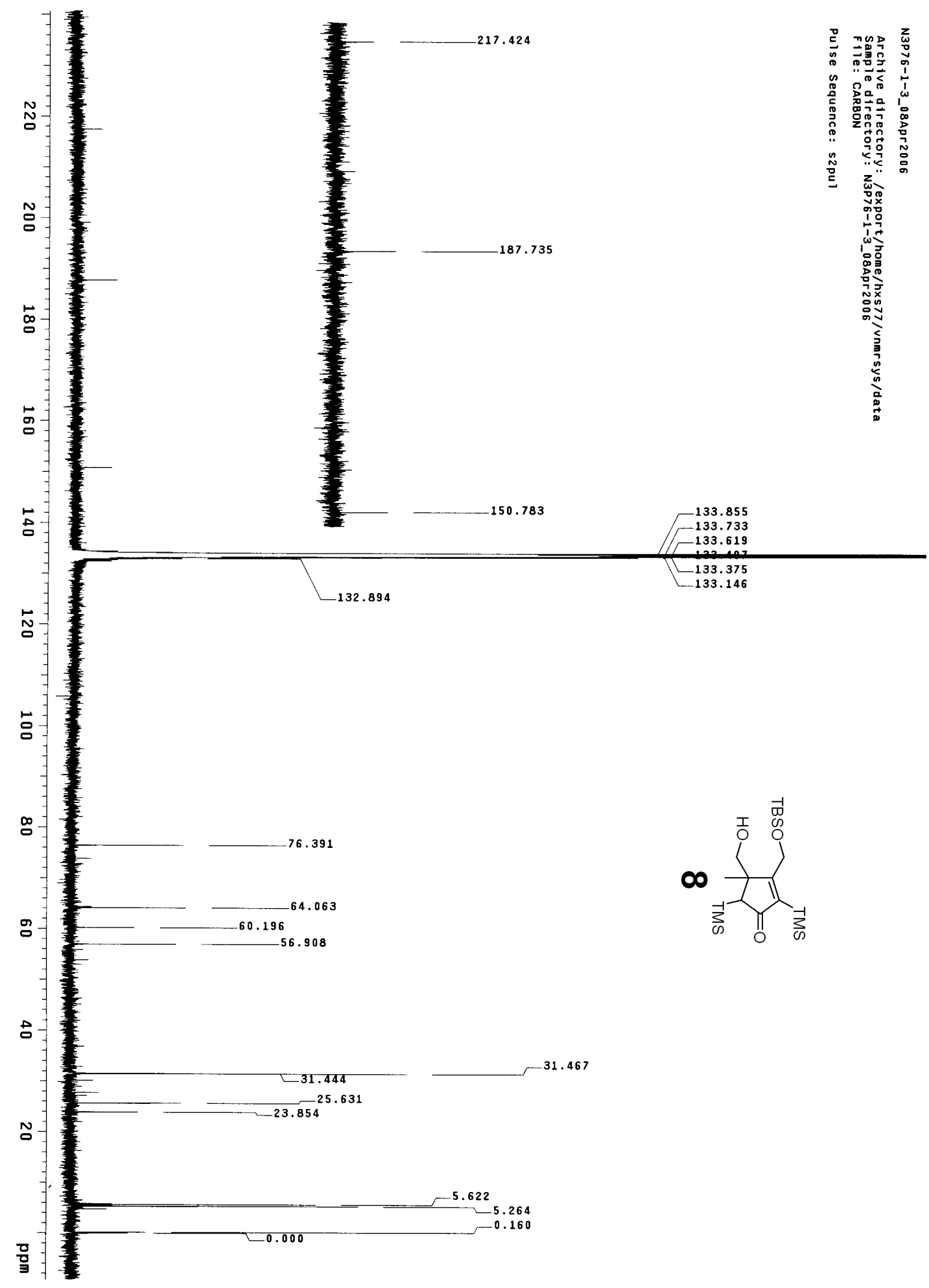




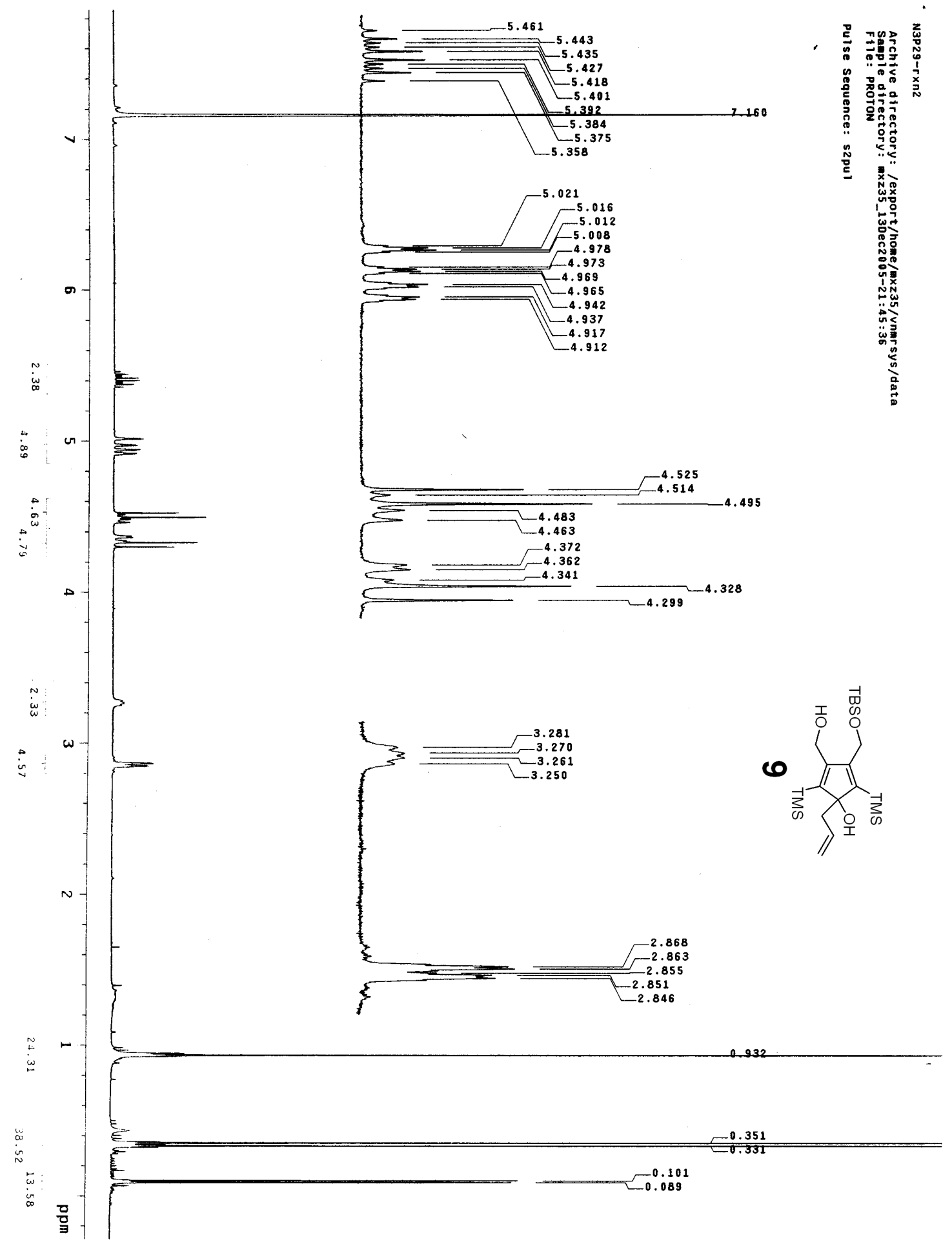




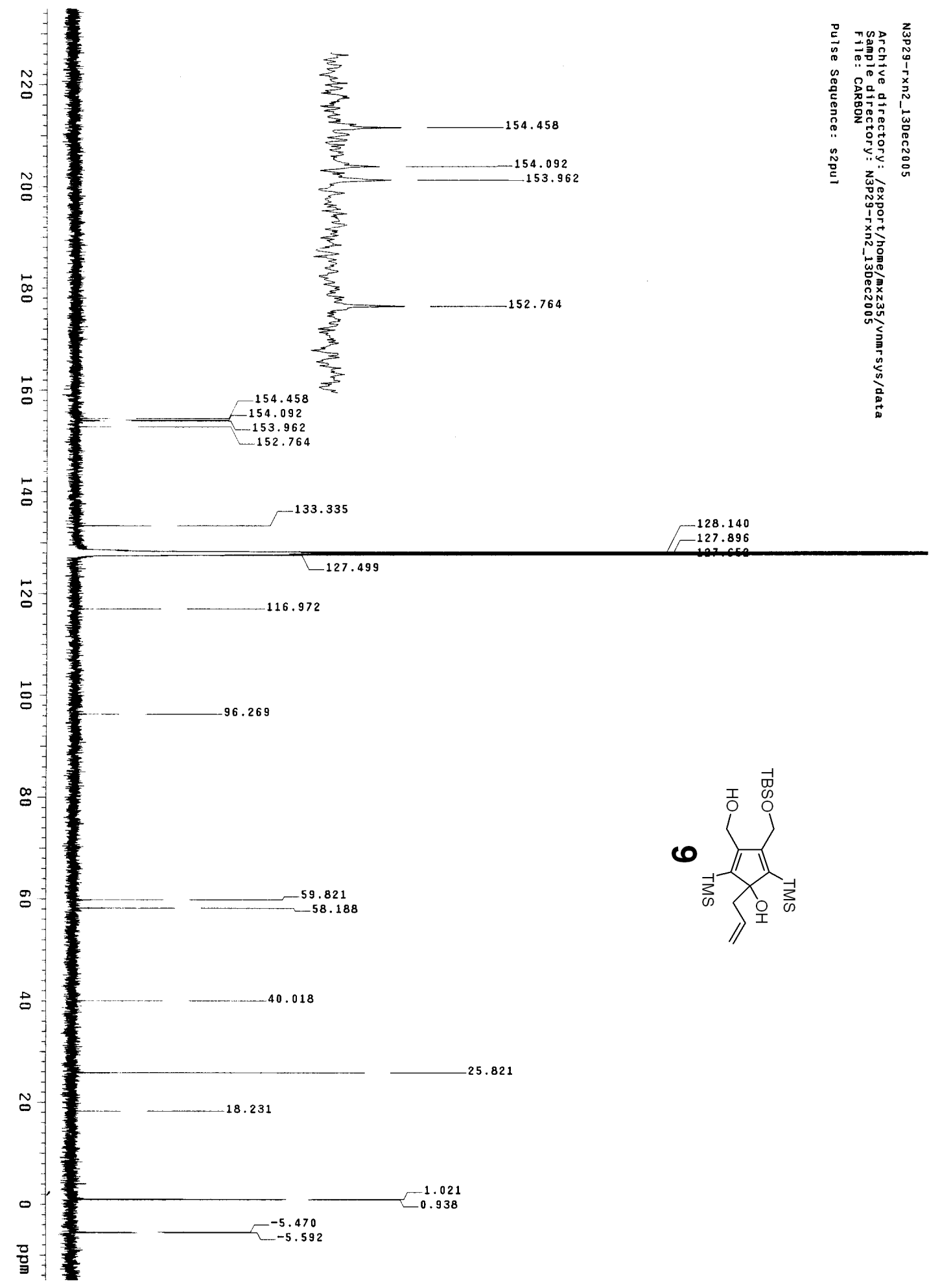




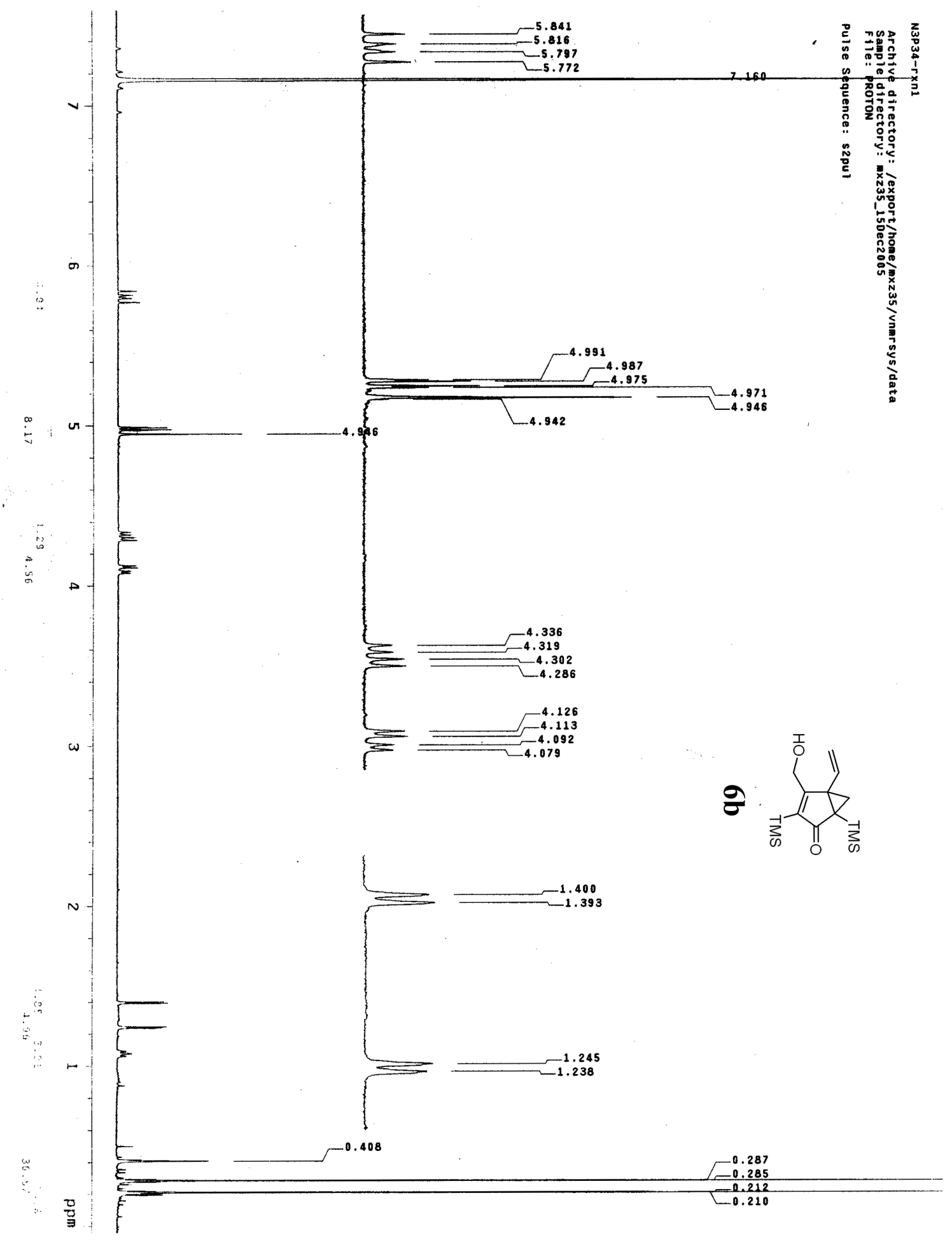




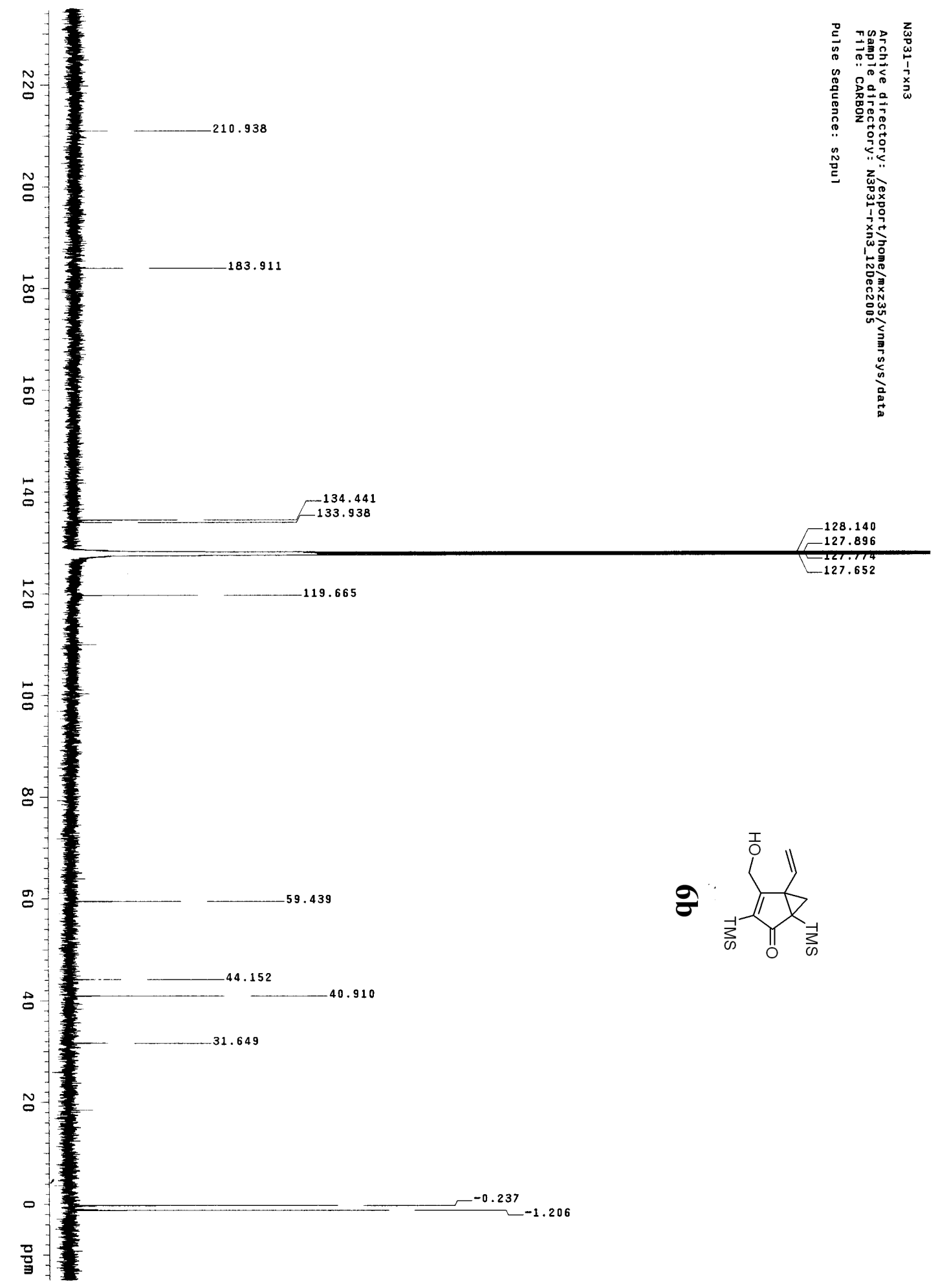




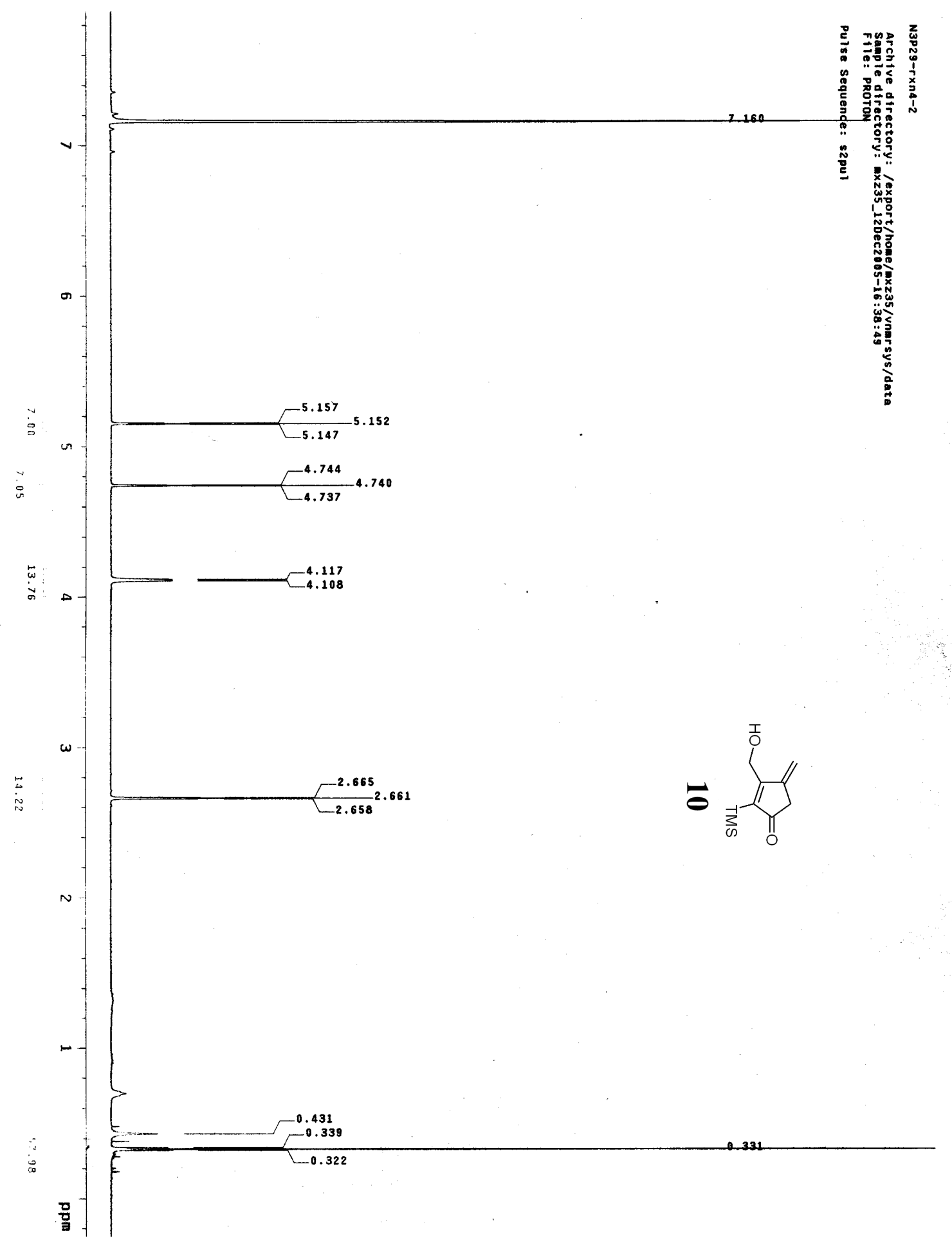




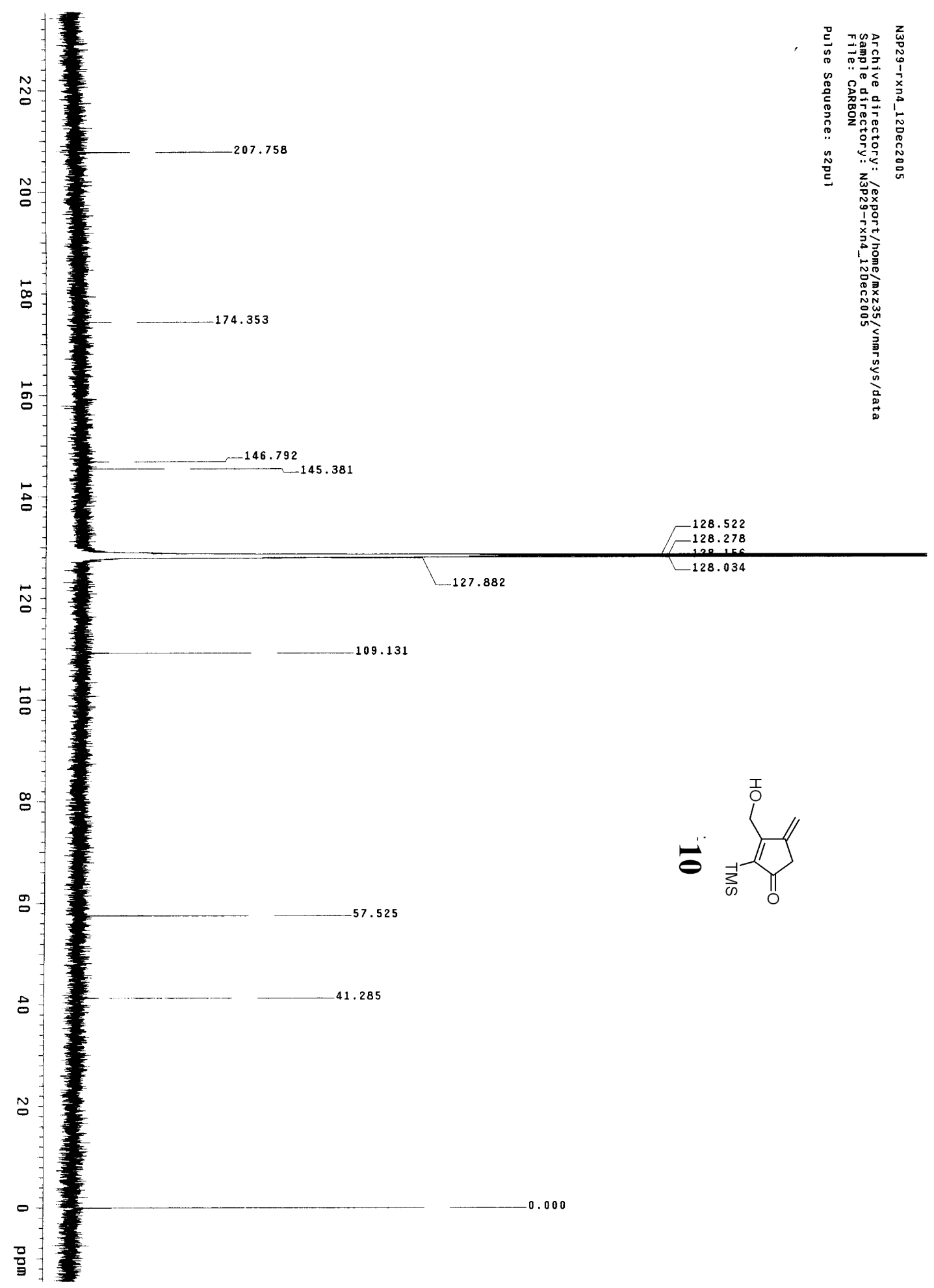




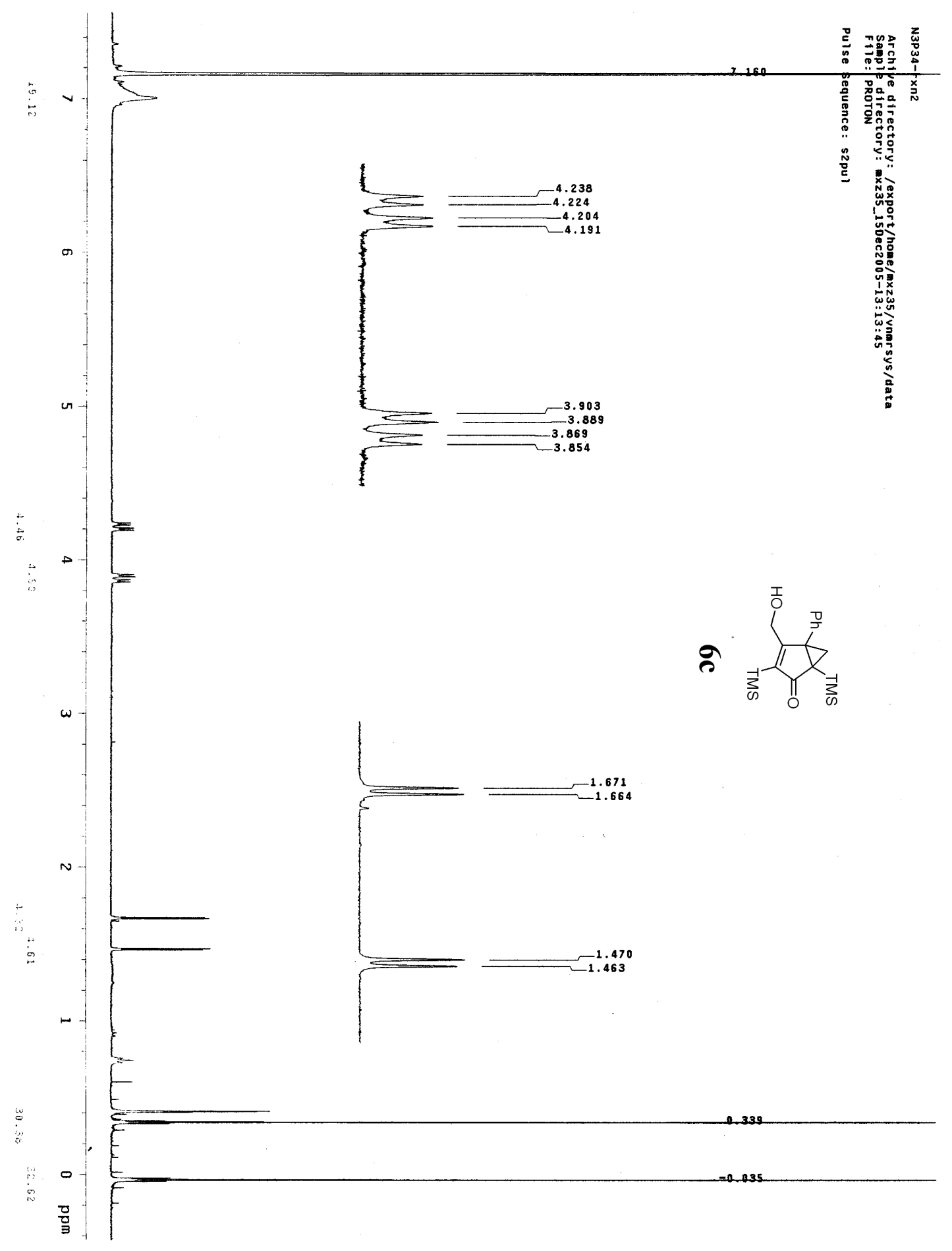




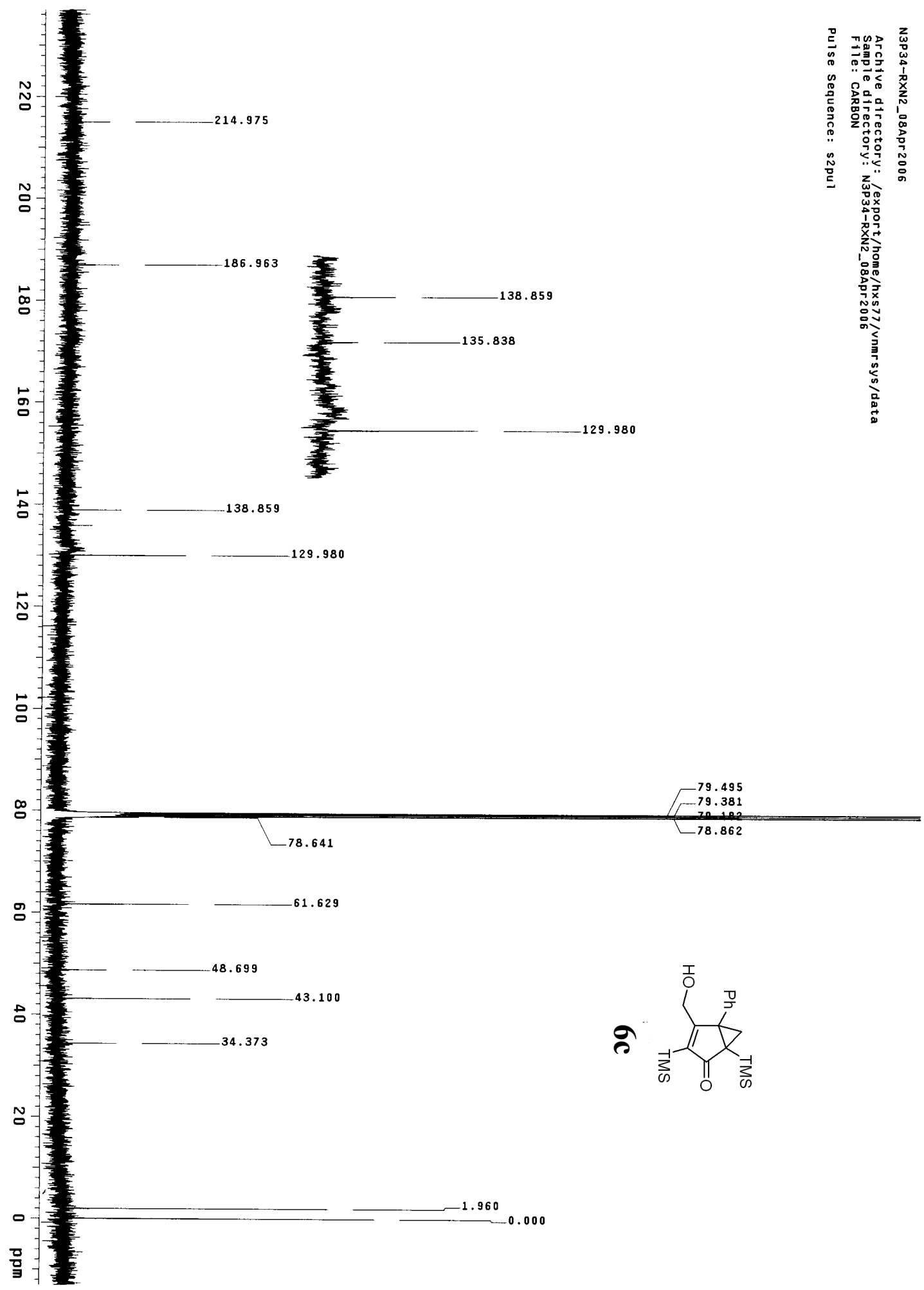




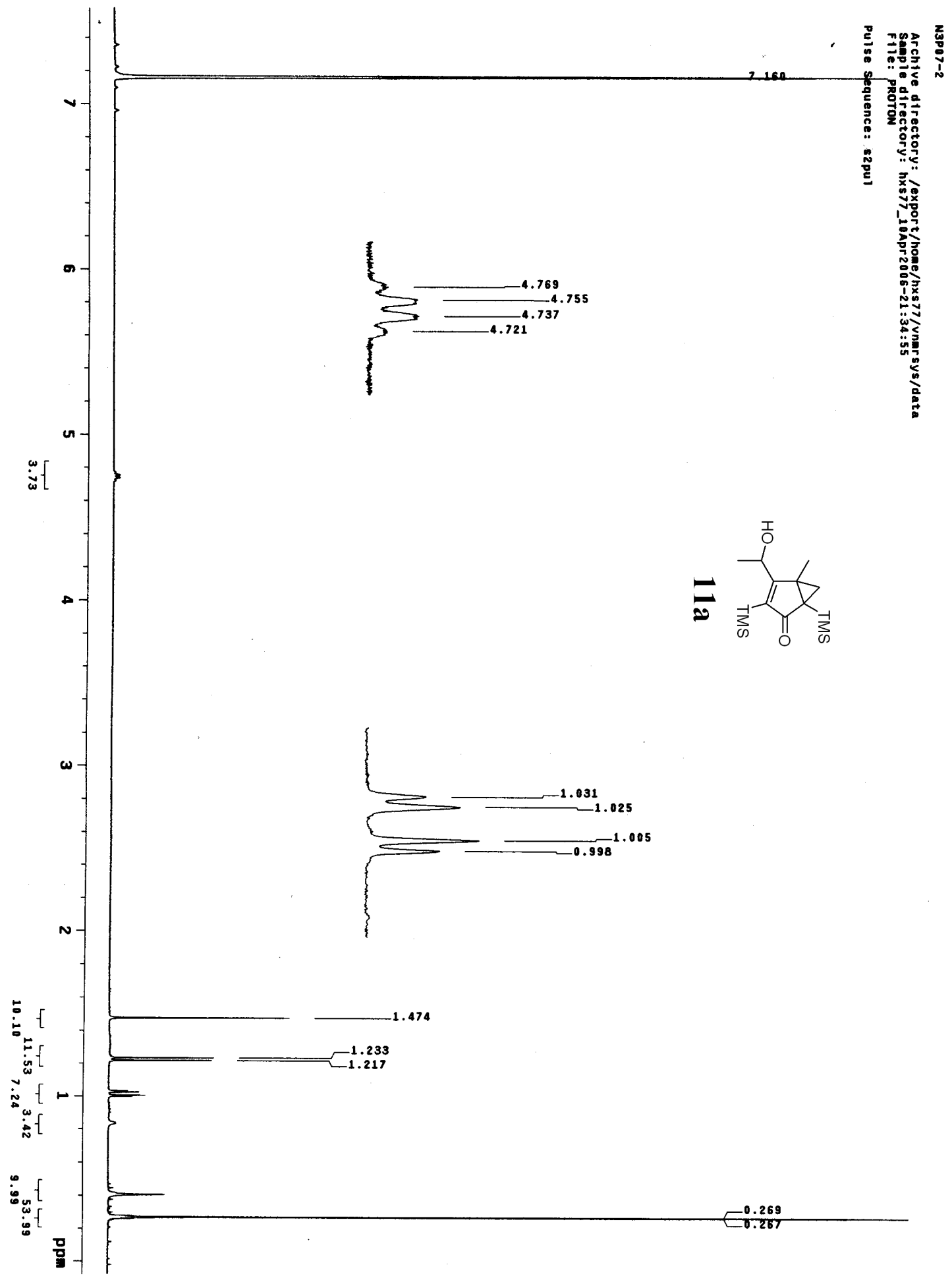




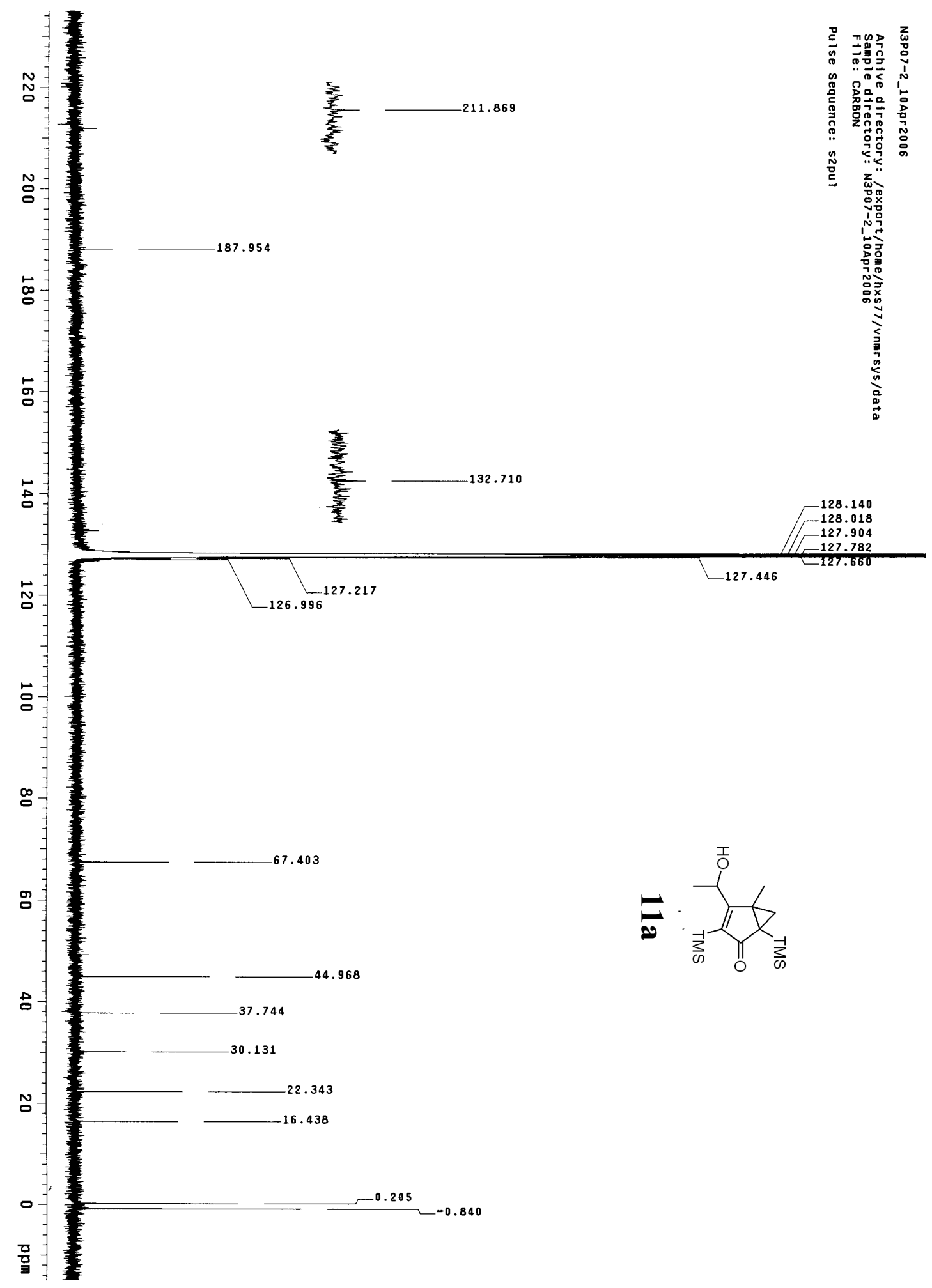




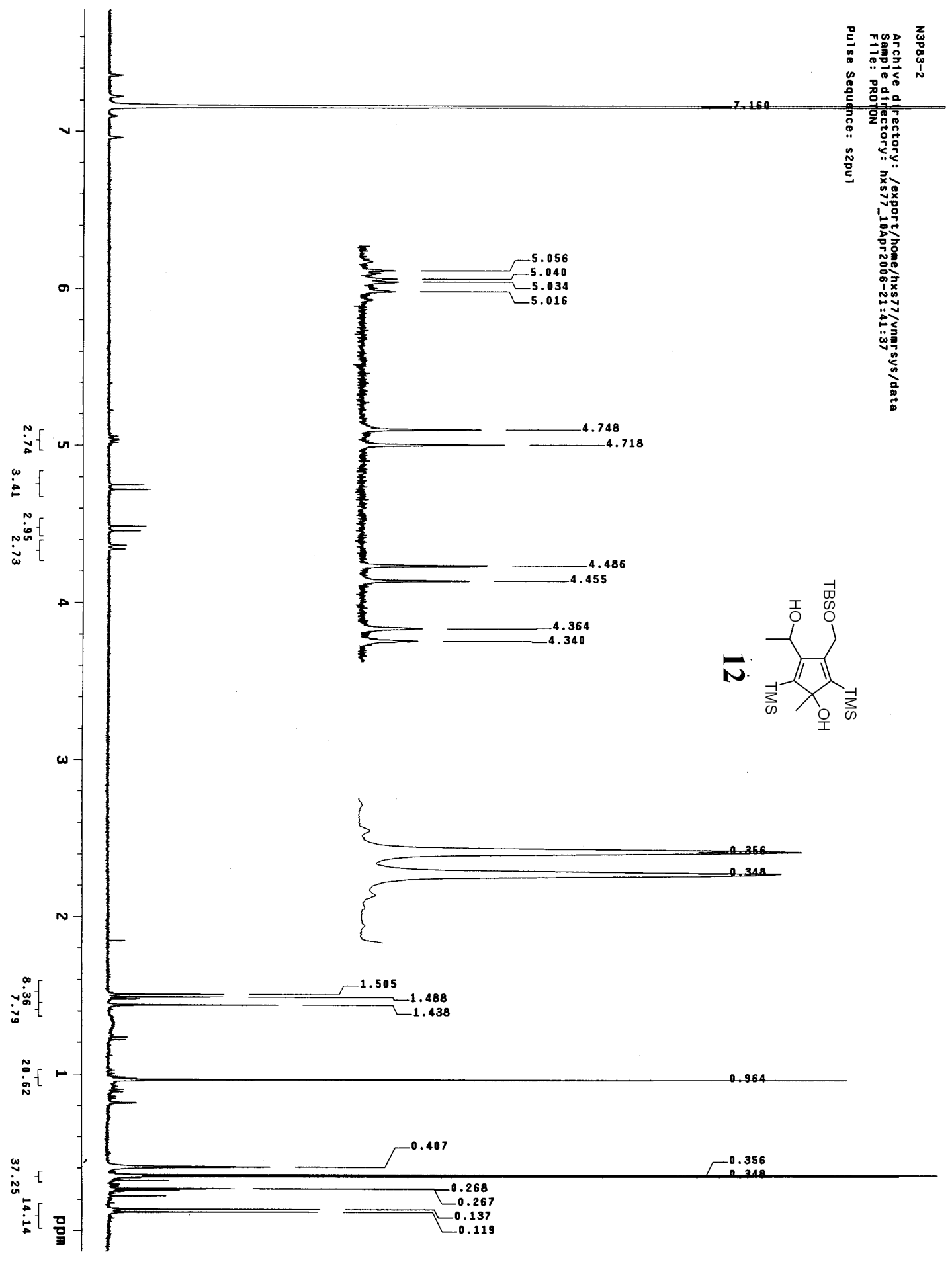




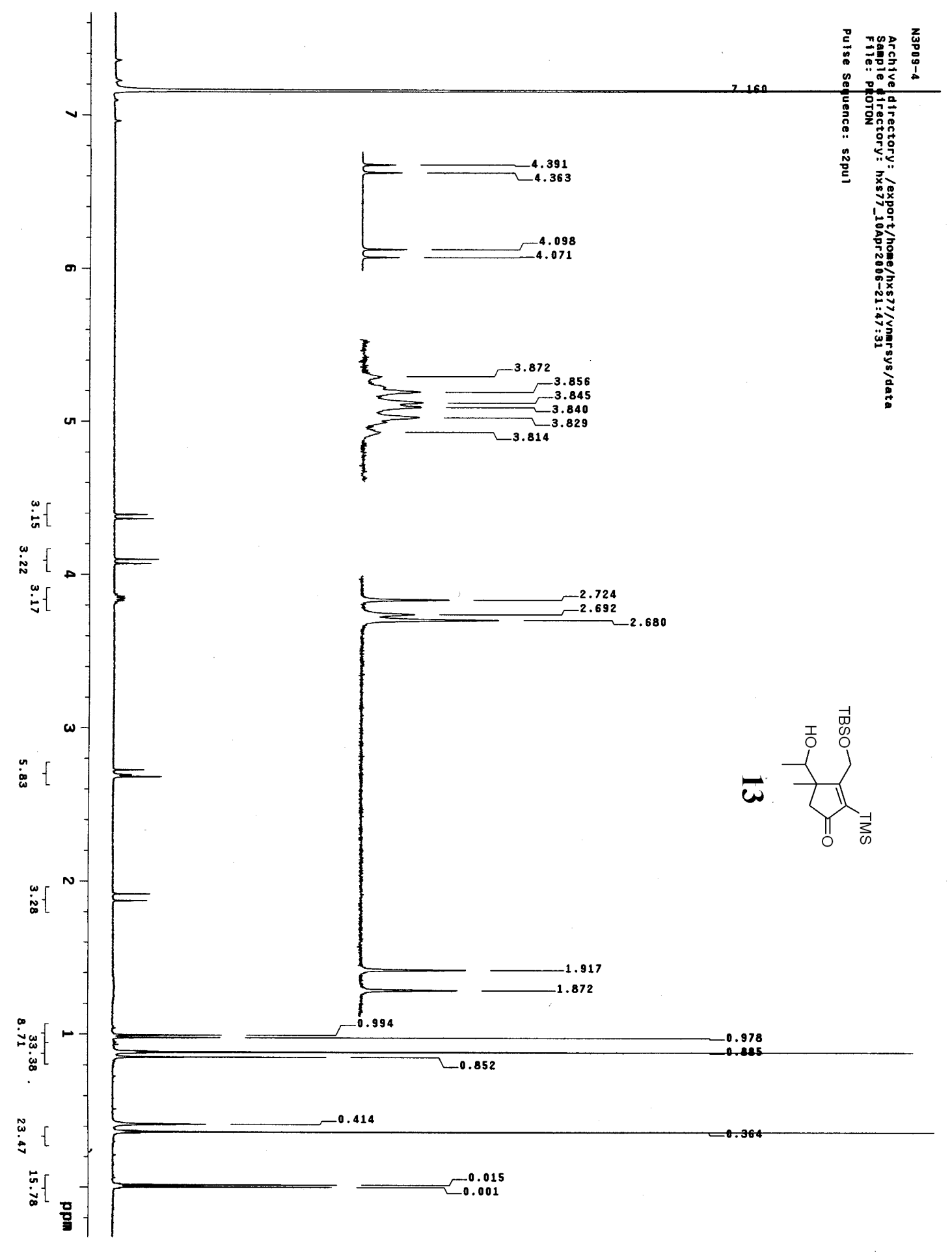




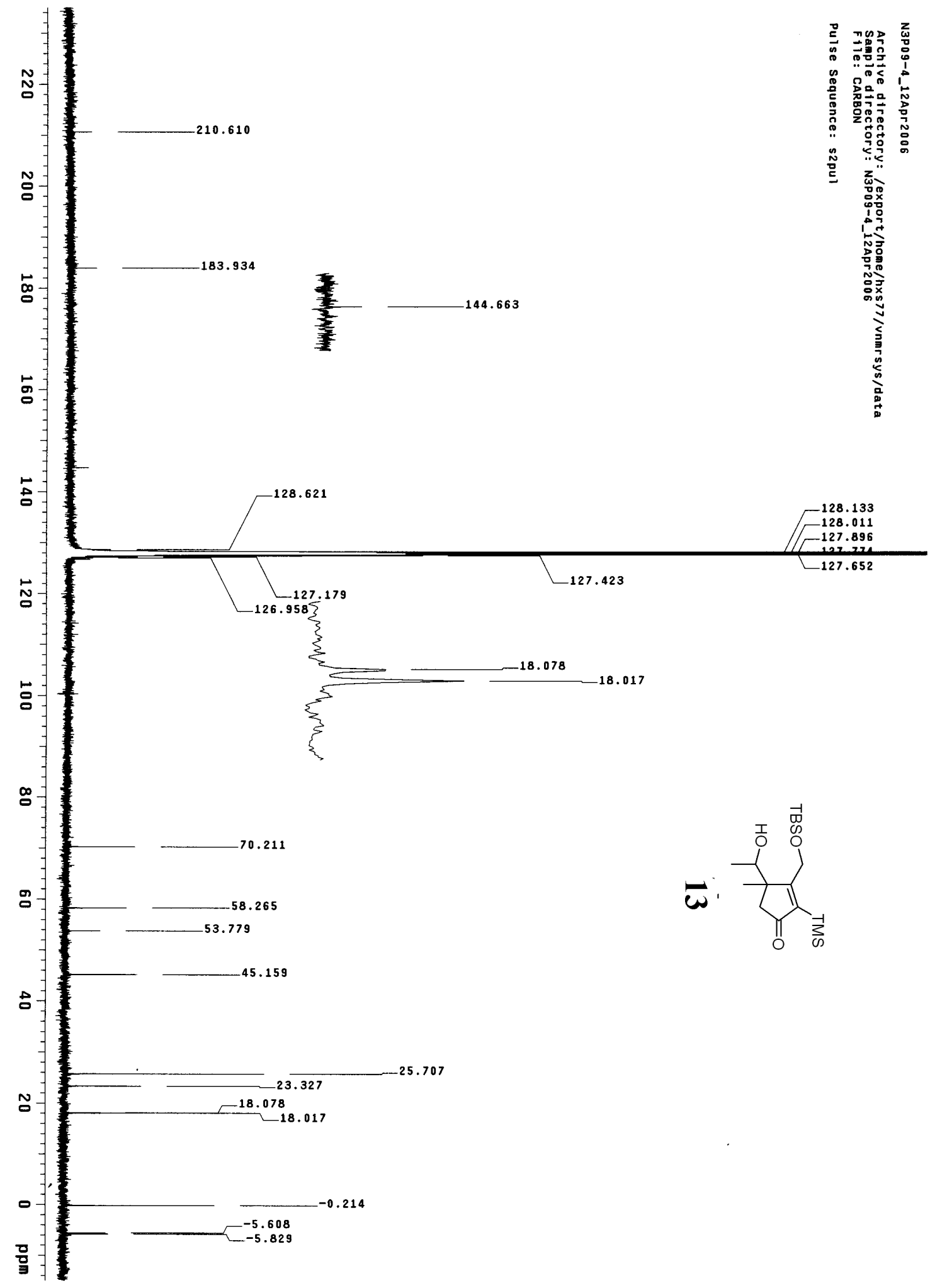




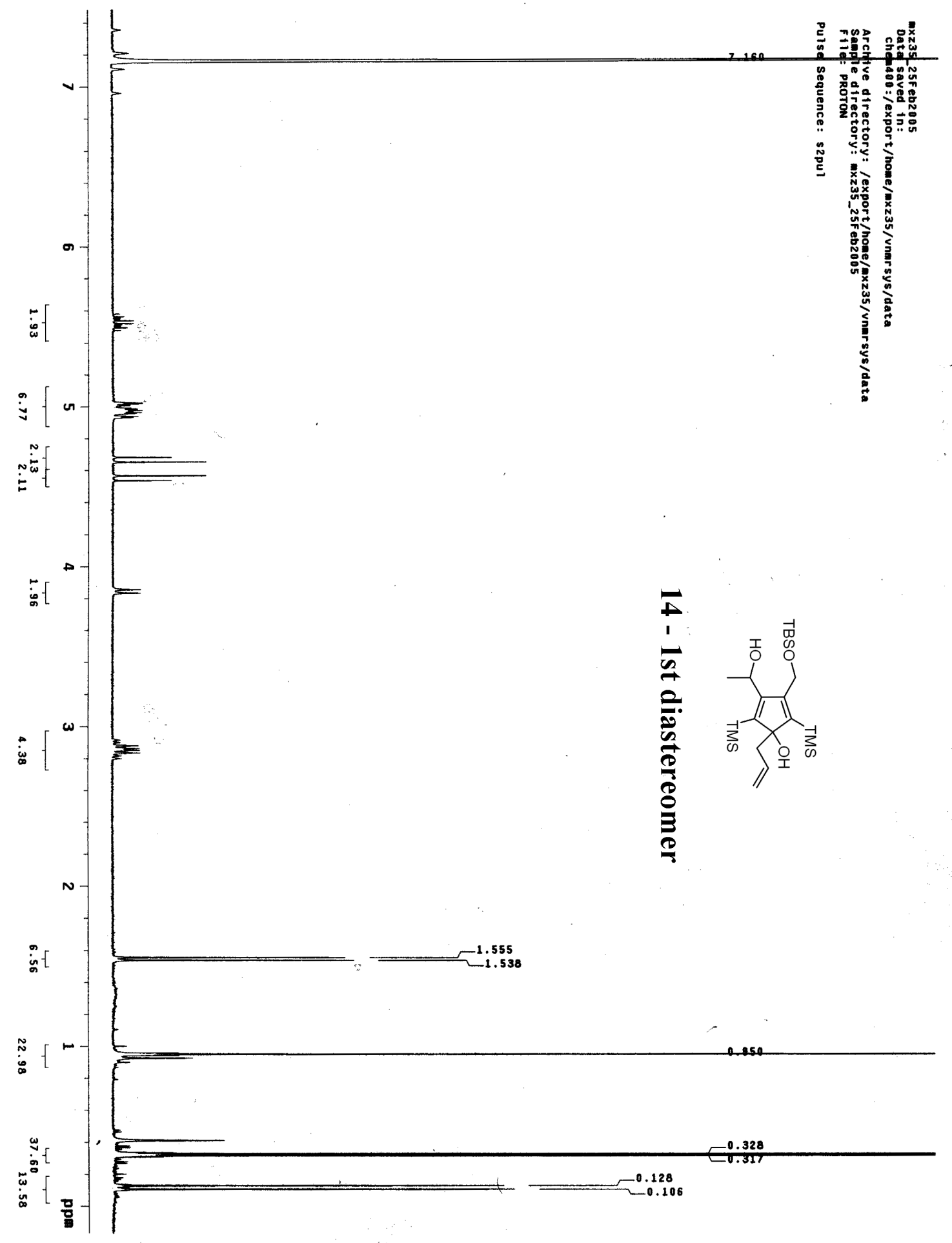




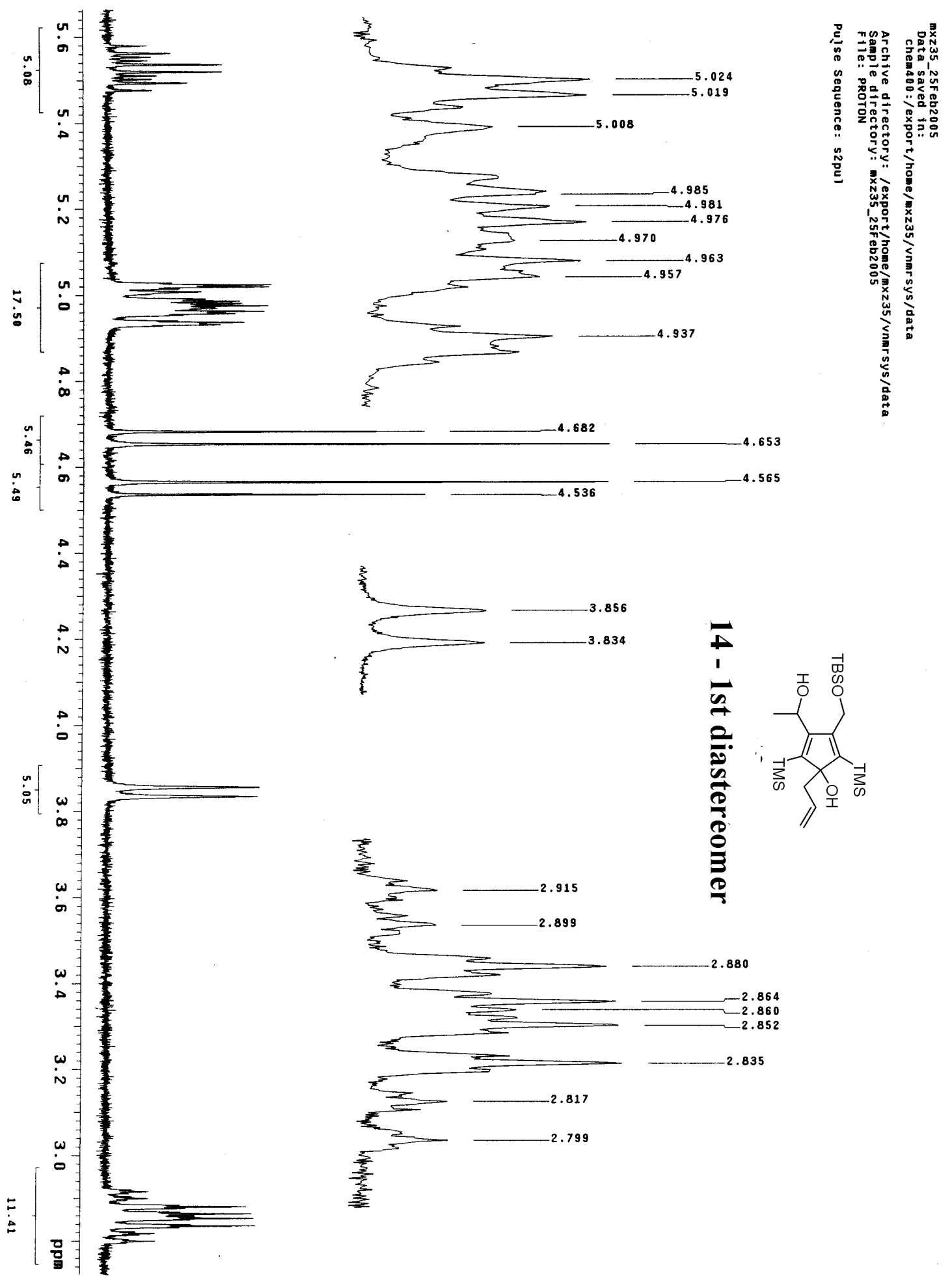




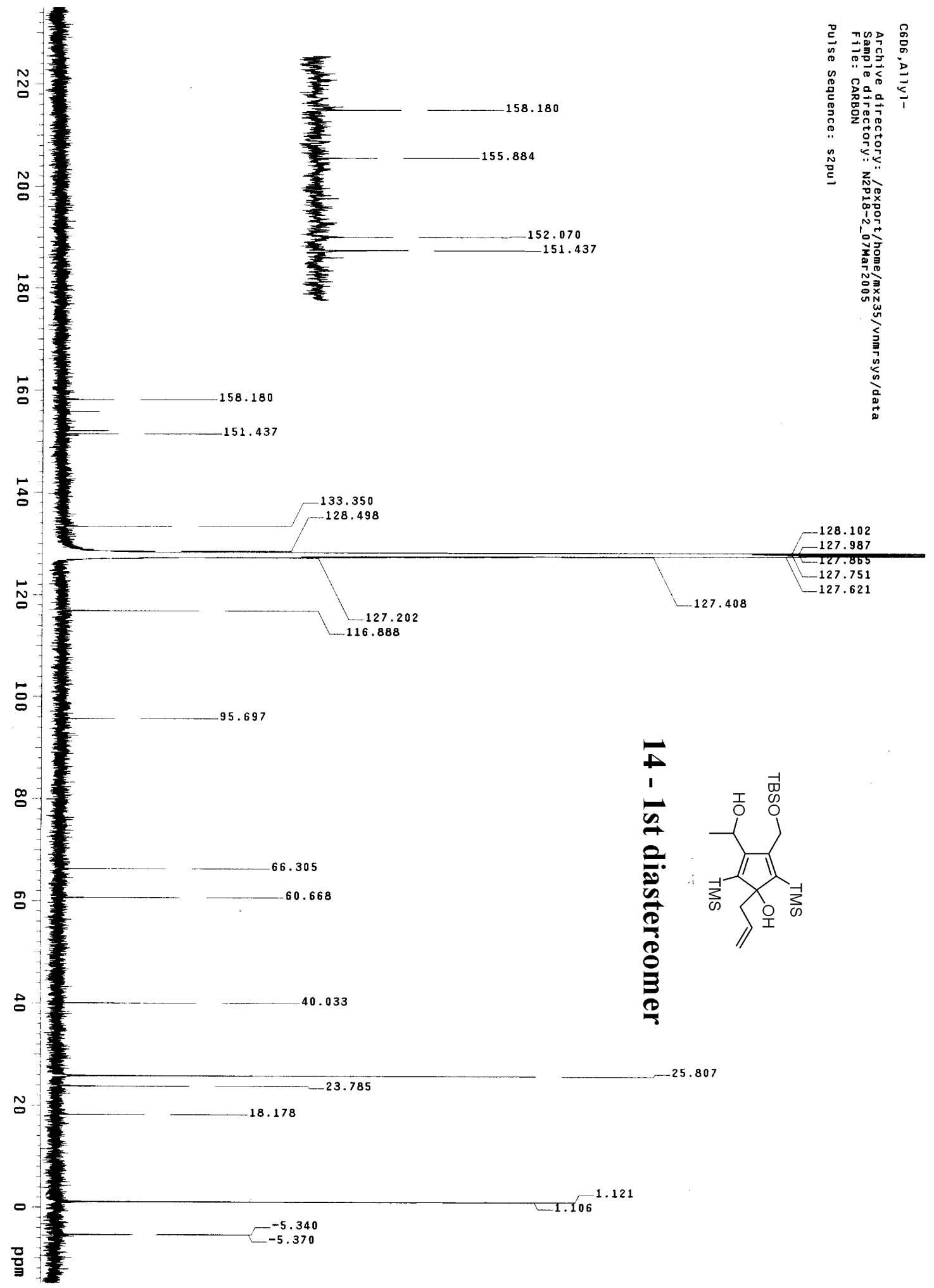




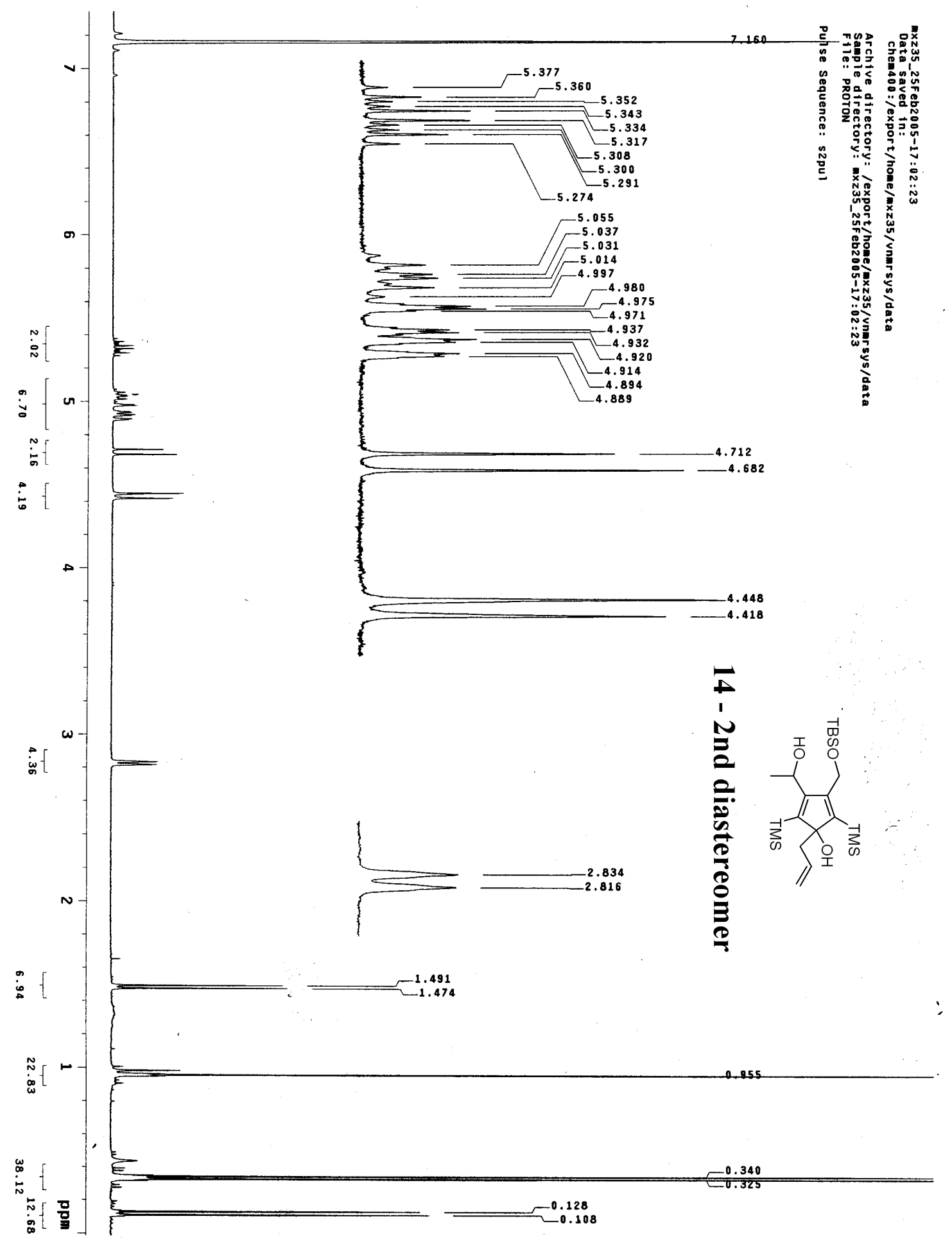




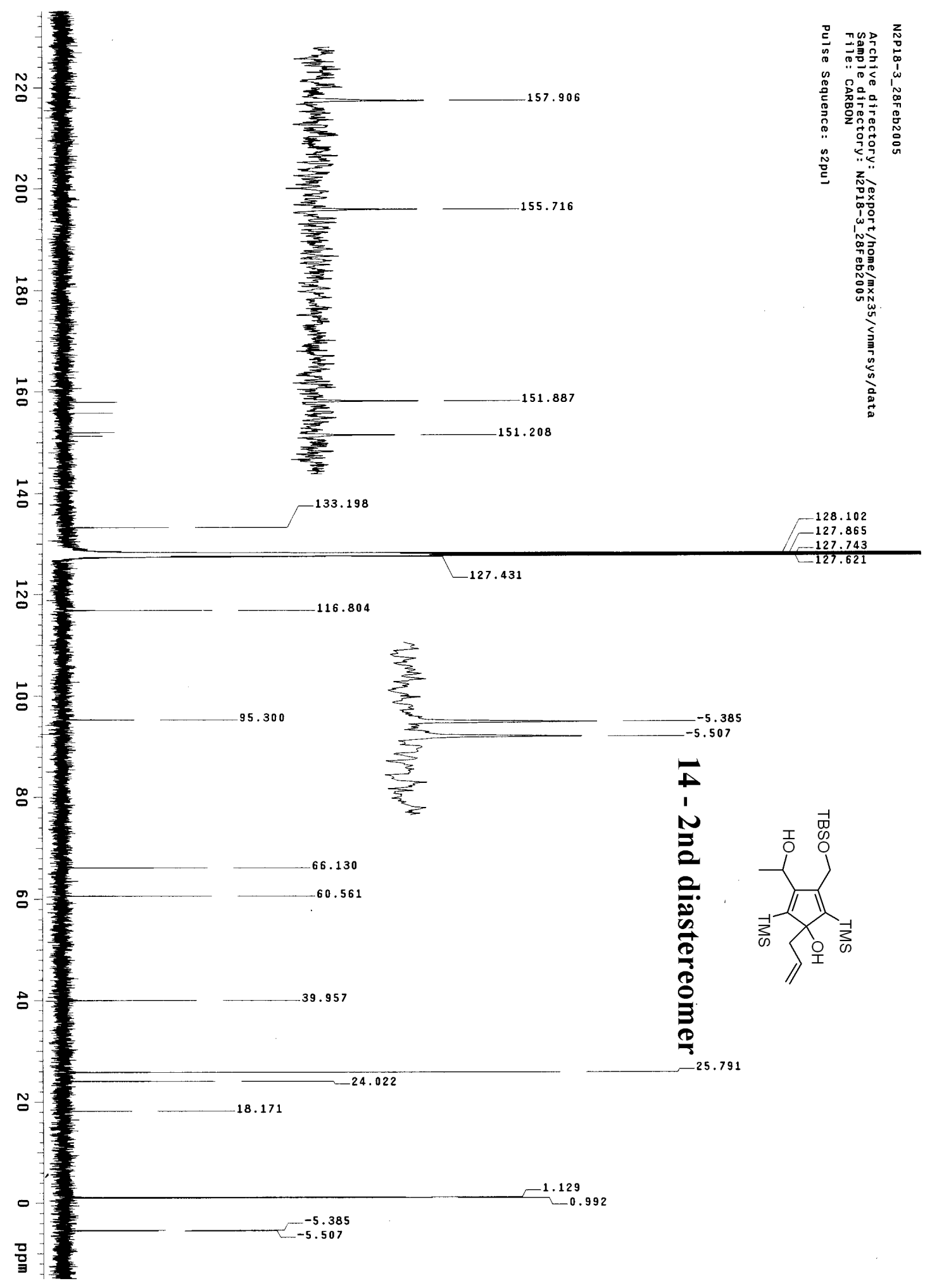




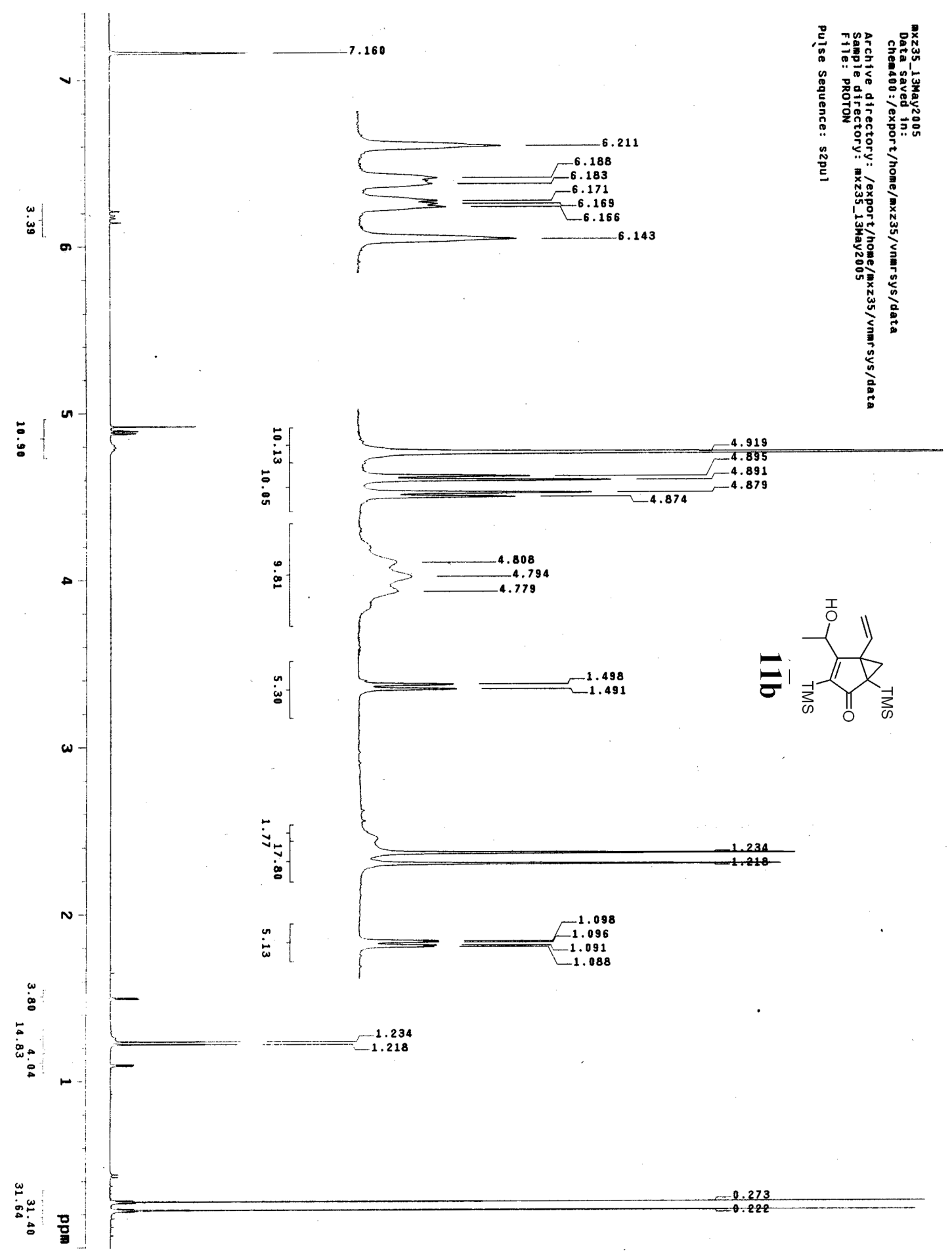




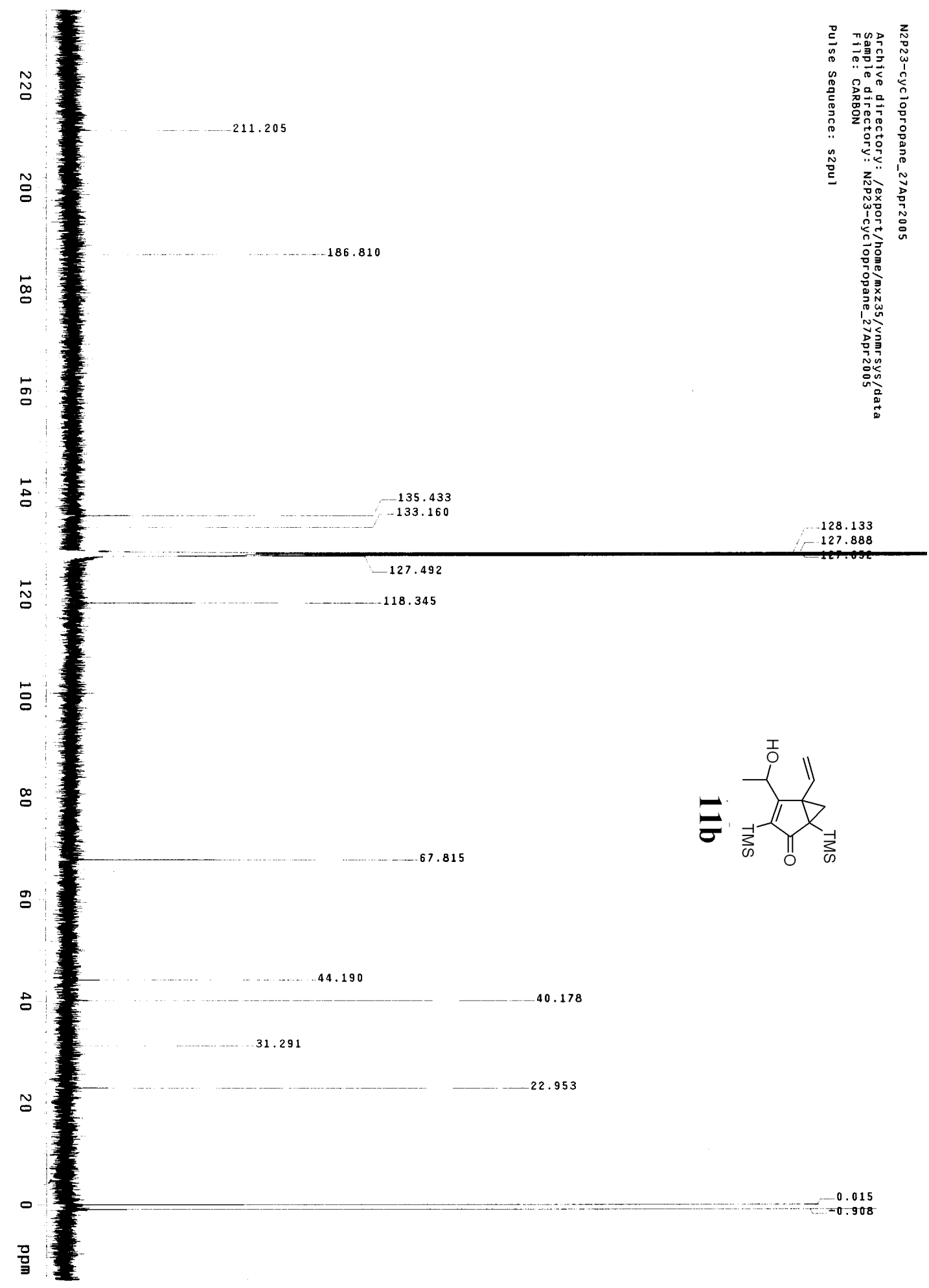



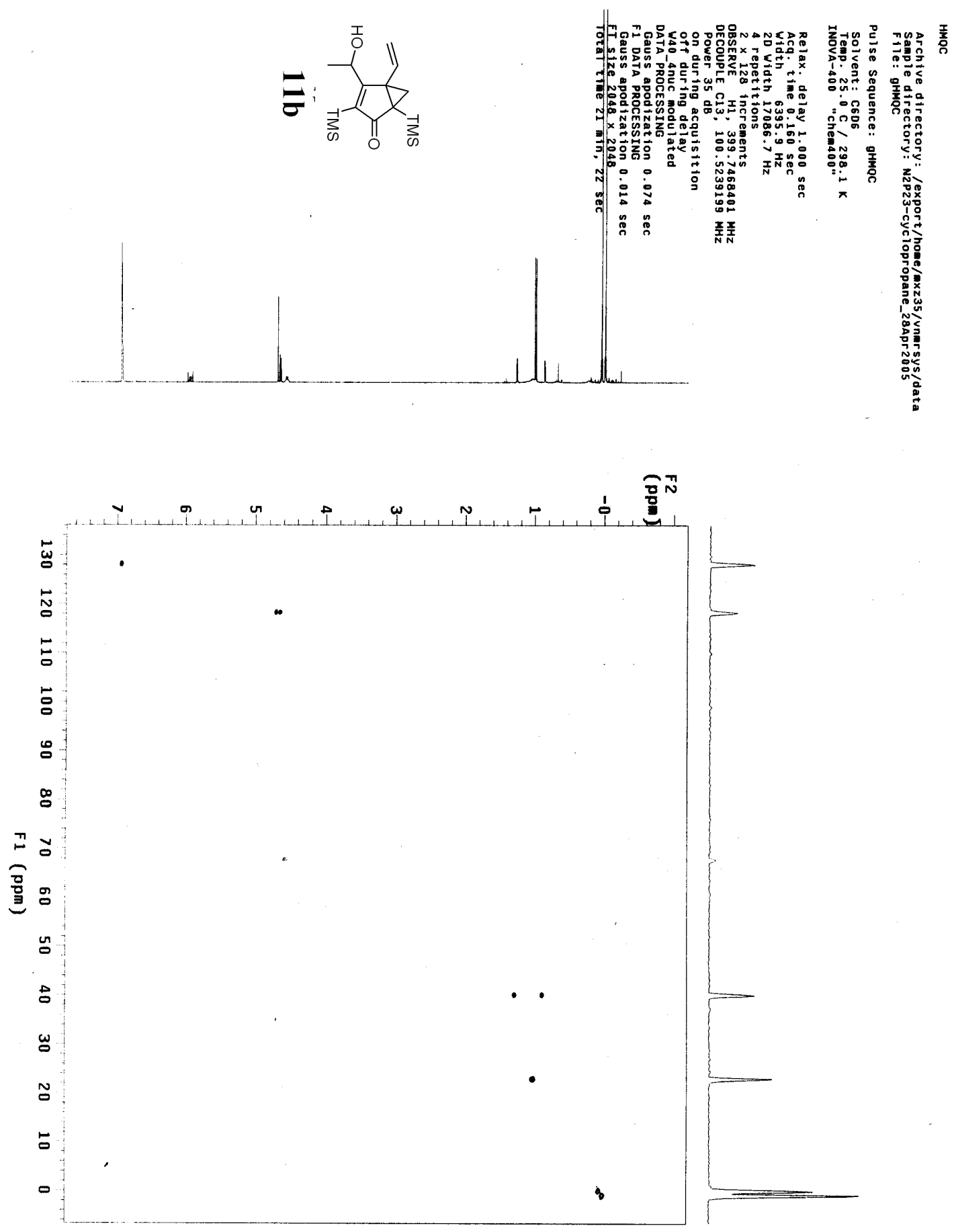

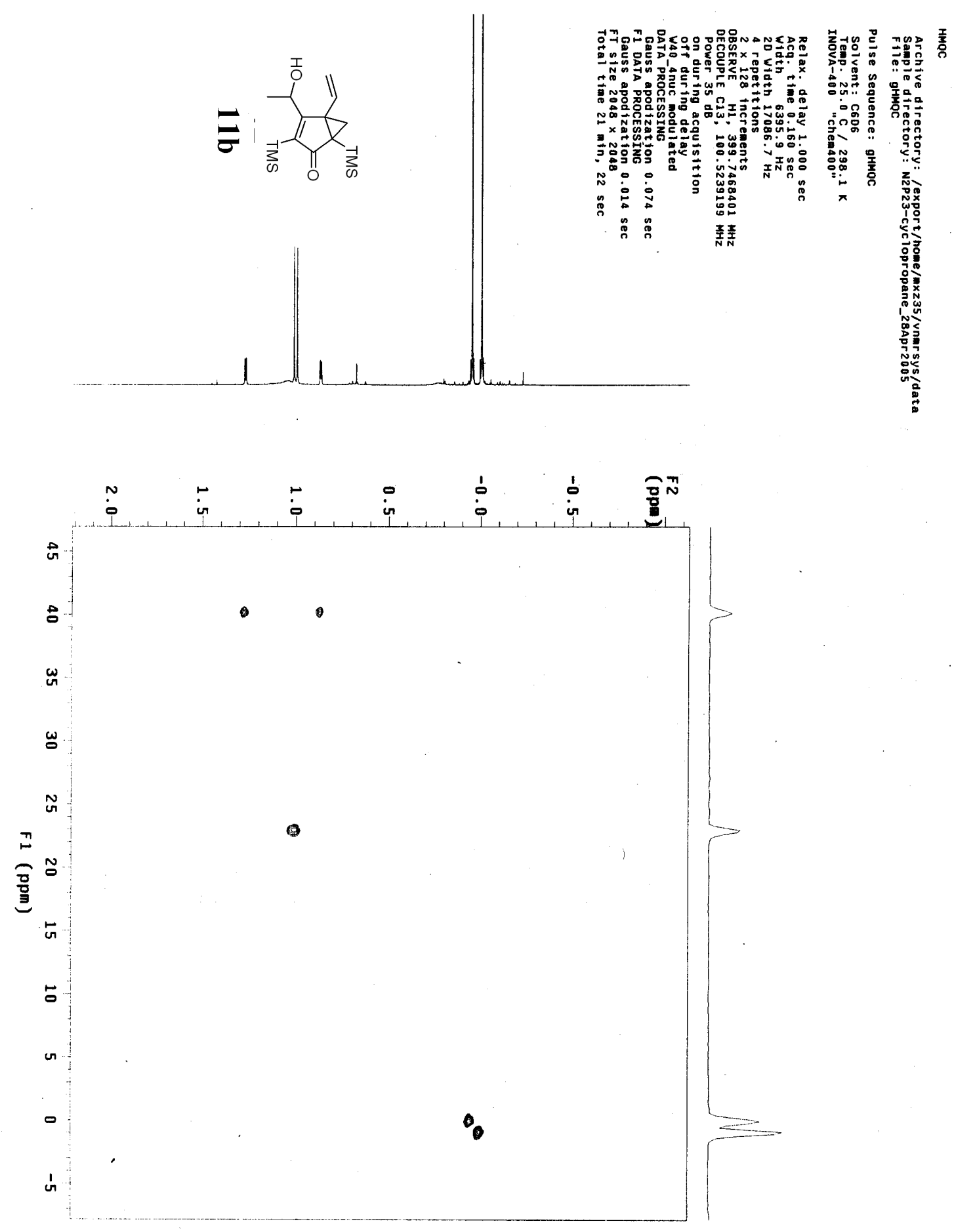

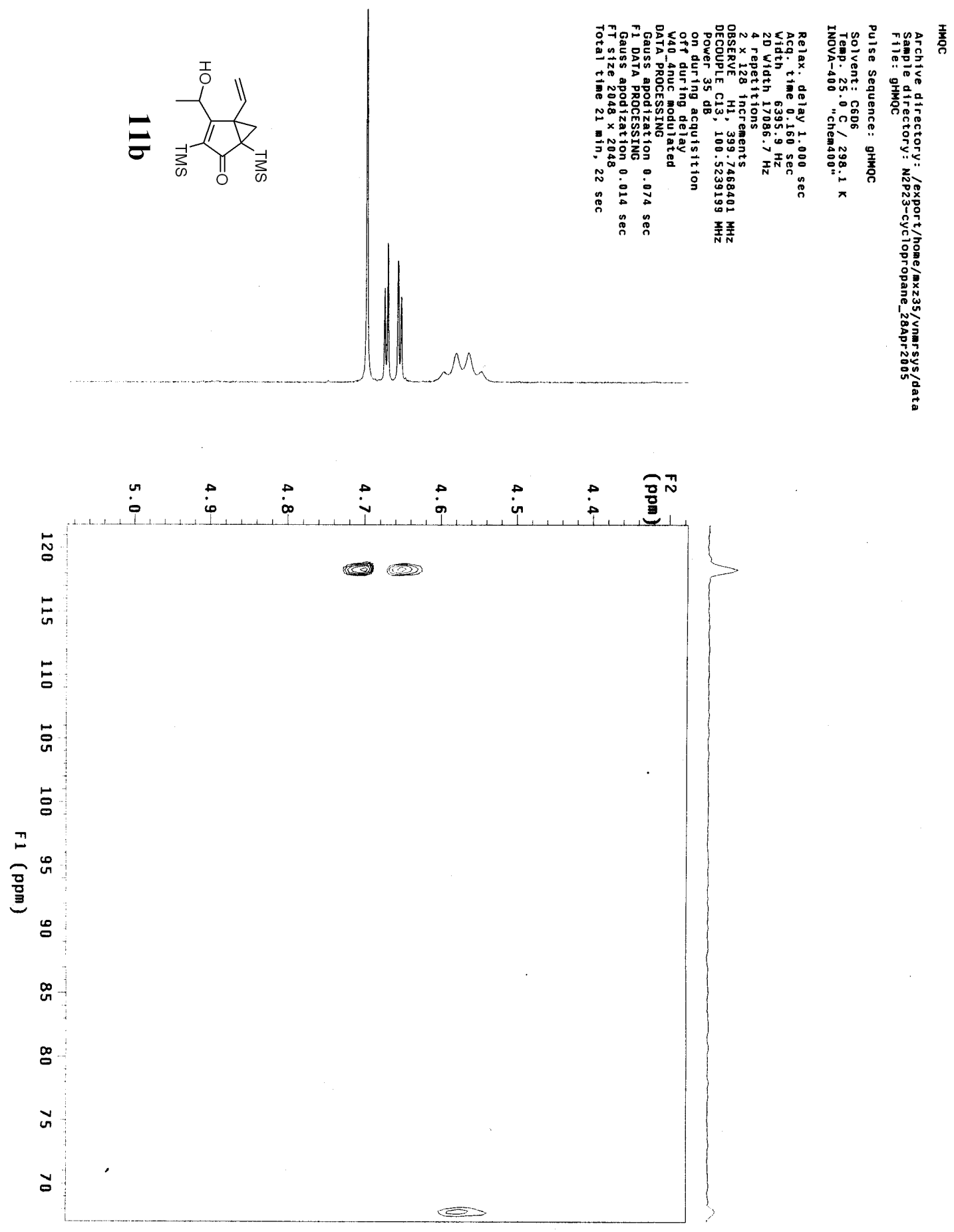


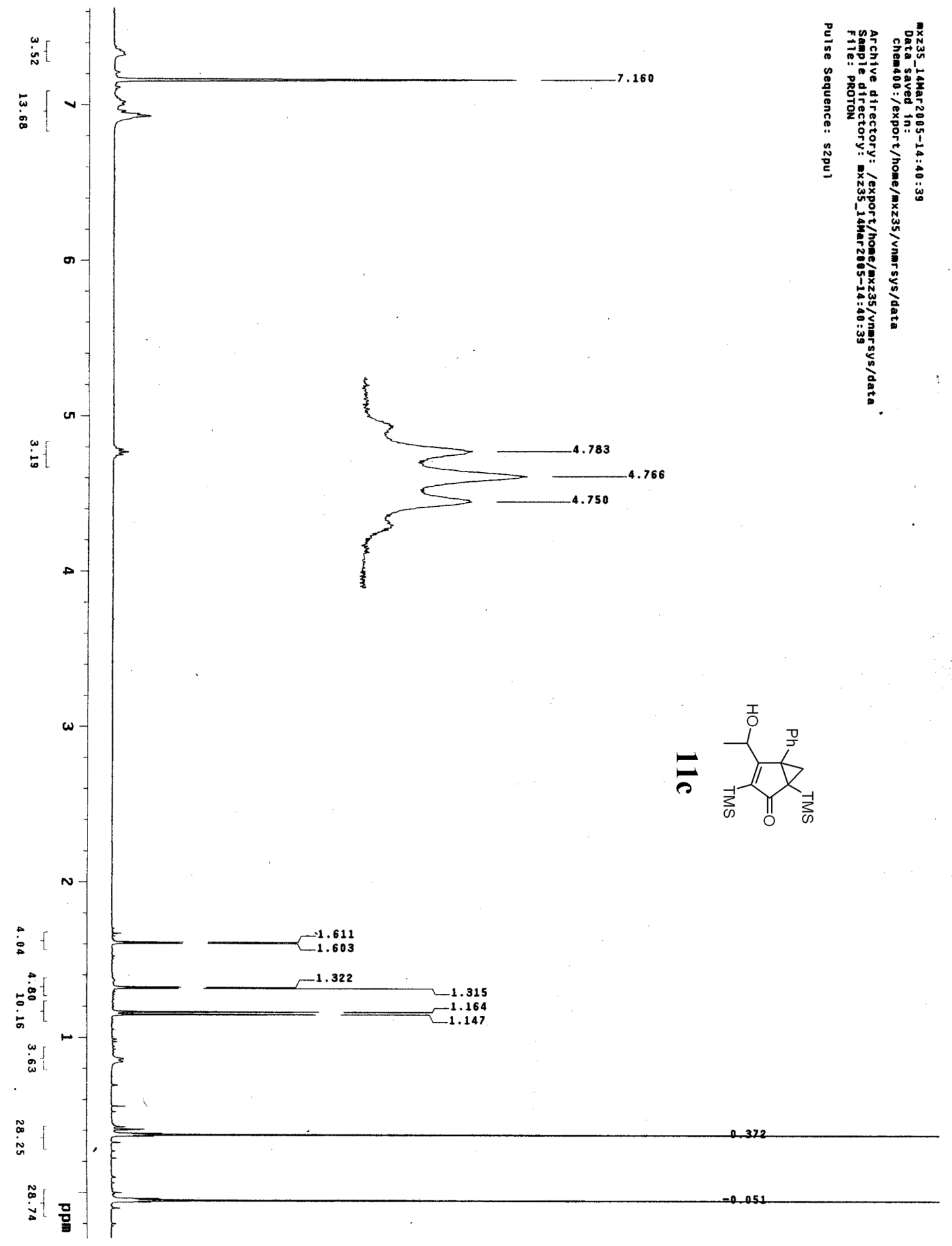




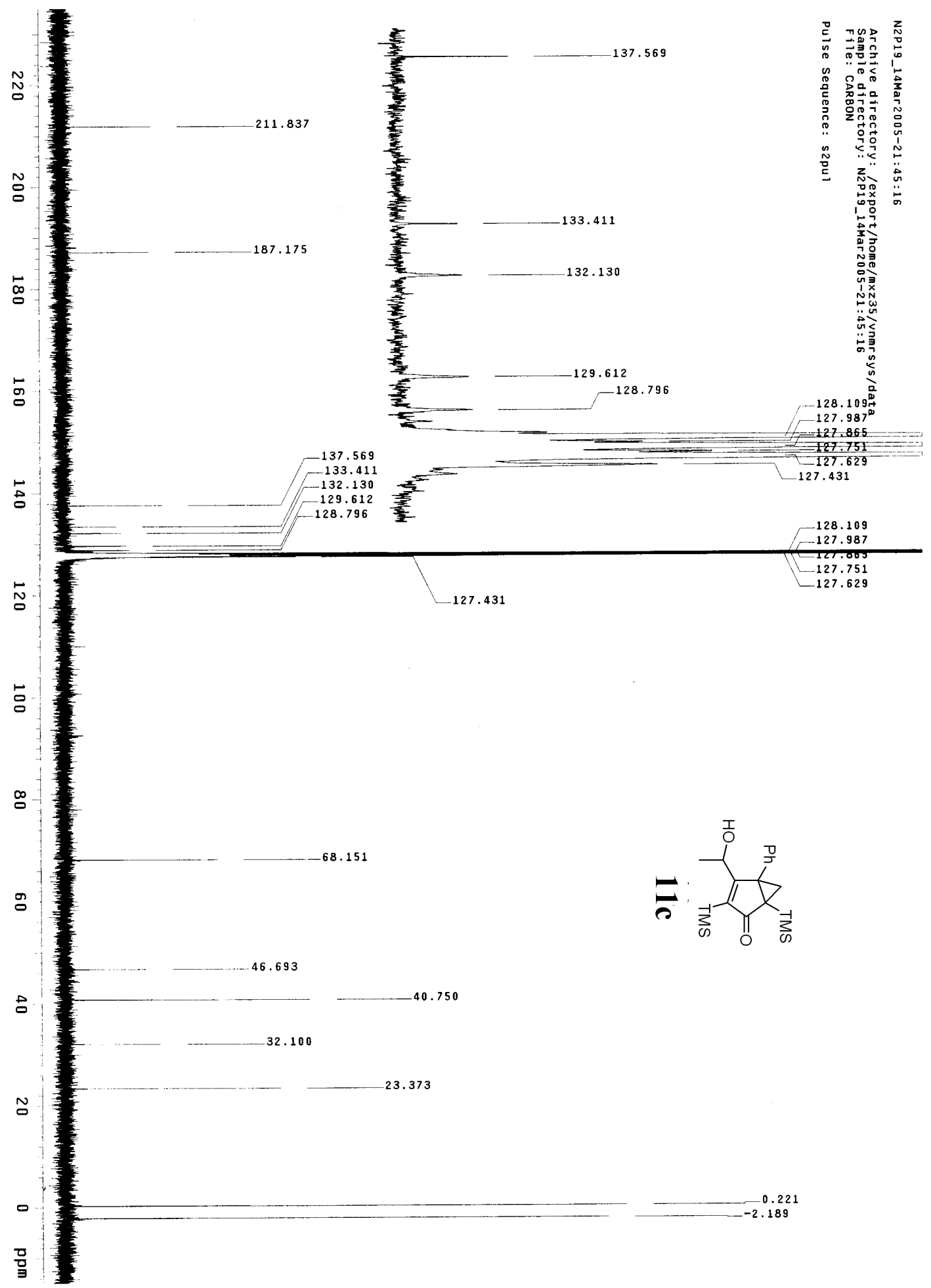




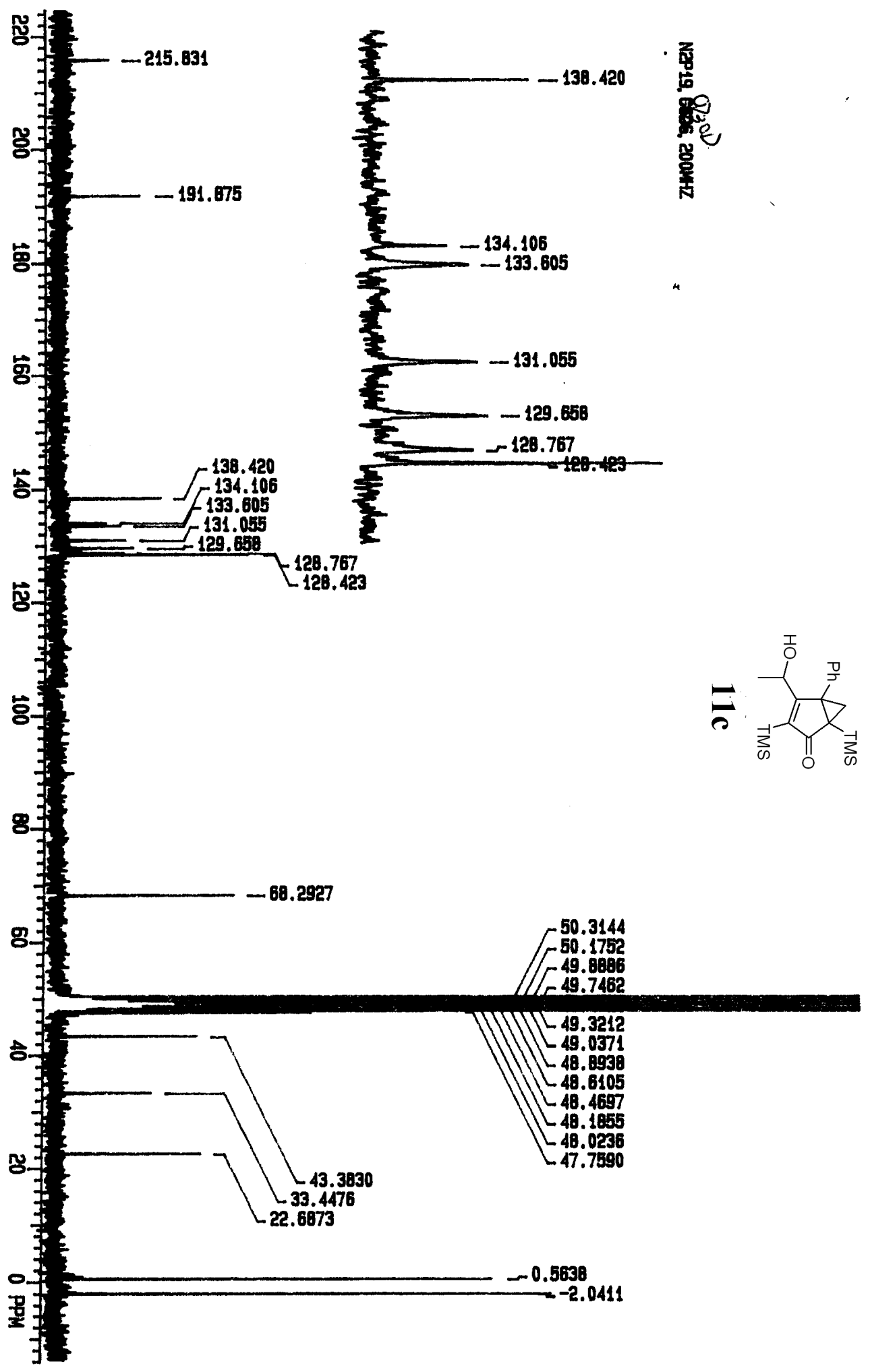



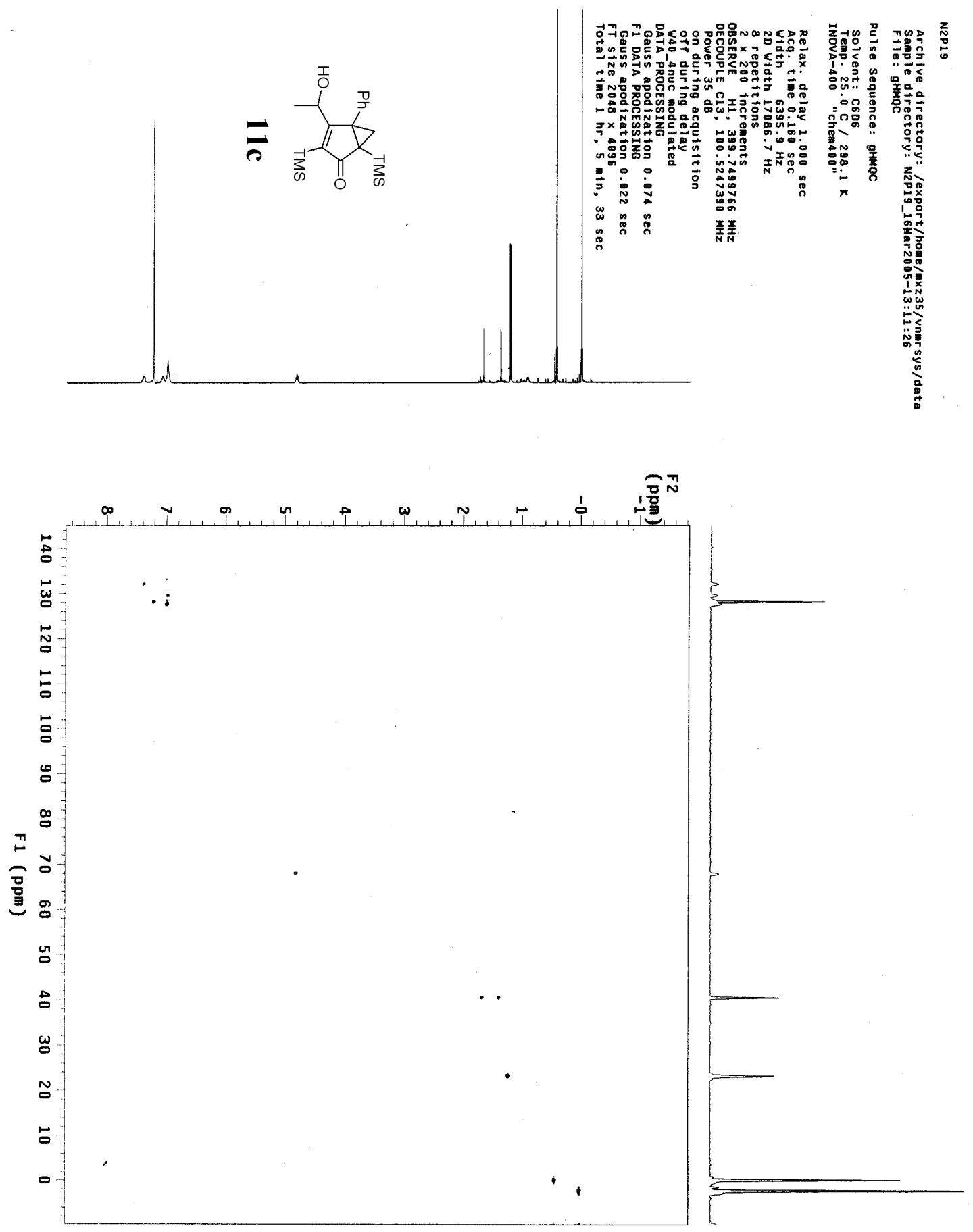

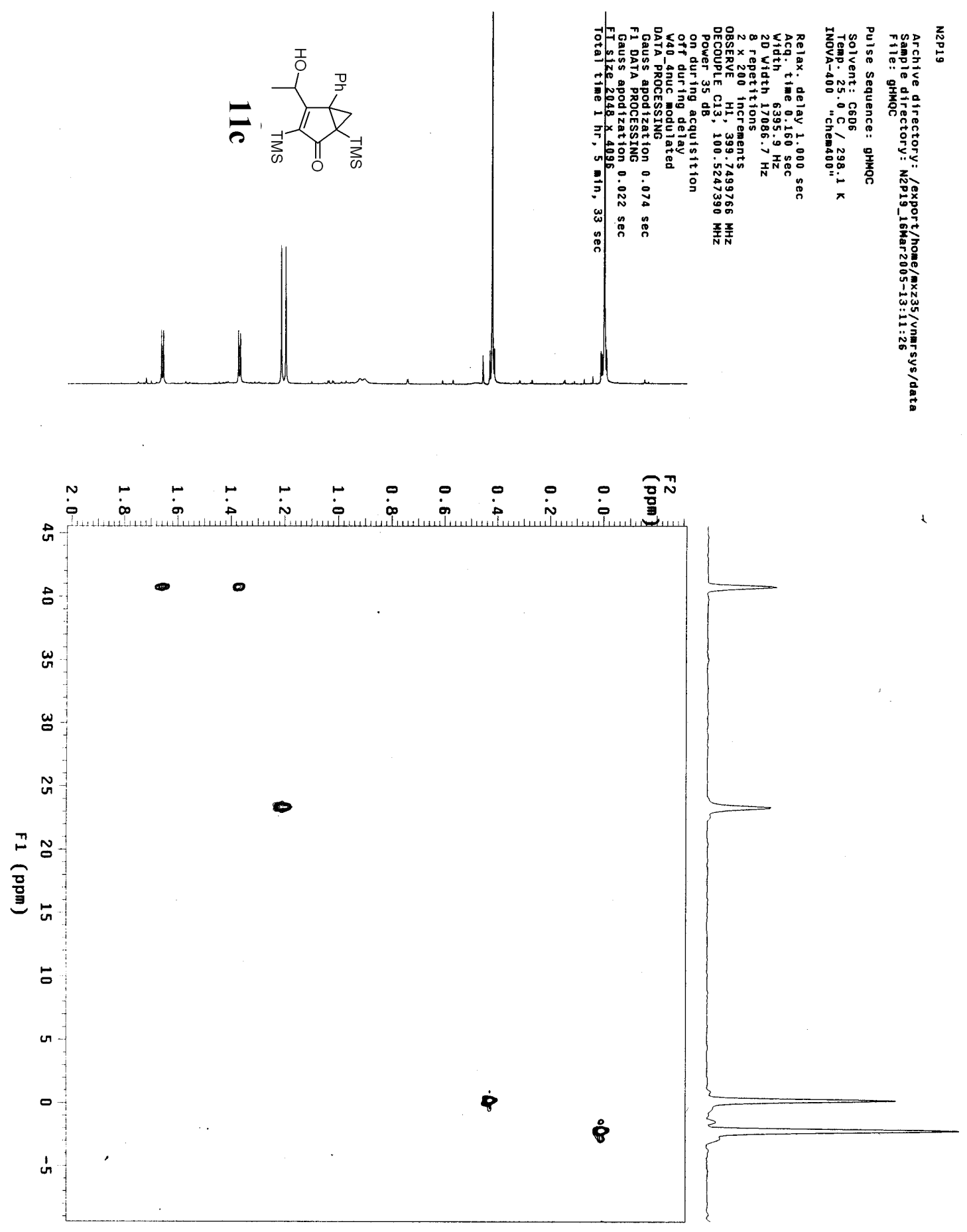

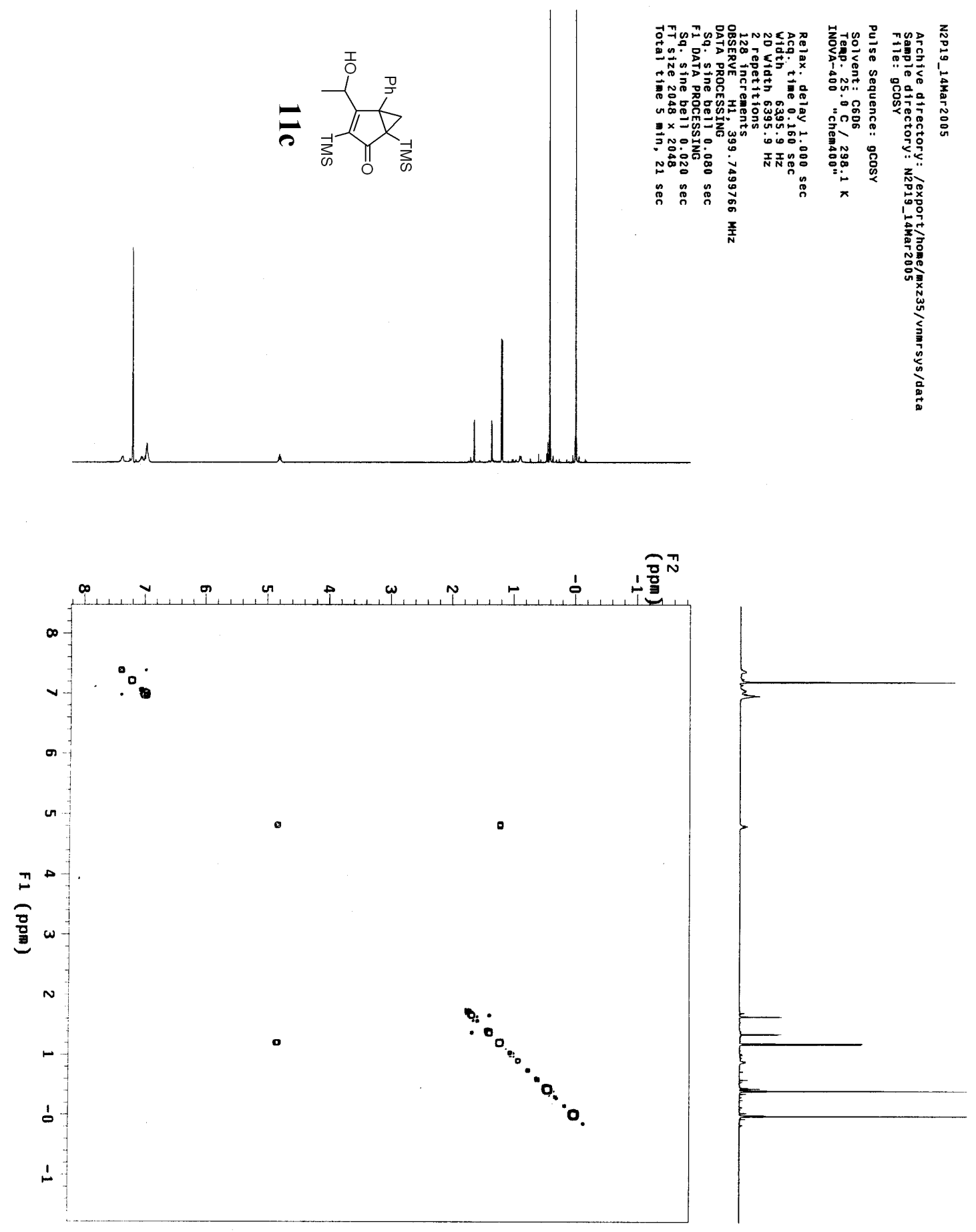
